\title{
Fractional proportional-resonant current controllers for voltage source converters
}

\author{
Daniel Heredero-Peris*, Cristian Chillón-Antón, Enric Sánchez-Sánchez, \\ Daniel Montesinos-Miracle
}

Centre d'Innovació Tecnologica en Convertidors Estàtics i Accionaments (CITCEA-UPC), Departament d'Enginyeria Elèctrica, Universitat Politècnica de Catalunya, ETS d'Enginyeria Industrial de Barcelona, Av. Diagonal, 647, Pl. 2, 08028 Barcelona, Spain

\section{A R T I C L E I N F O}

\section{Keywords:}

Control

Current control

Frequency domain analysis

Harmonic analysis

\begin{abstract}
A B S T R A C T
This paper proposes a novel fractional proportional-resonant controller, which applies fractional order calculus to the well-known proportional-resonant controllers. The focus of the study is the current control loop of voltage source converters. The main merit of the proposed fractional controller formulation lies into the use of fractional exponents in the integro-derivative parts obtaining a controller with an extra degree of freedom. This degree of freedom allows the phase delay to be improved for a wide frequency range in comparison with the conventional proportional-resonant controllers. Furthermore, the obtained controller results in a lower order transfer function that reduces the computational burden when multiple current frequencies have to be tracked. As fractional integro-derivative exponents are not directly implementable, five mathematical approaches are explored, selecting the Chareff's approximation for the fractional controller operator's implementation. A tuning procedure for such a controller is also addressed. The new controller formulation is validated in a $20 \mathrm{kVA}$ laboratory set-up based on a silicon-carbide converter, and it is implemented in a DSP. Two AC output converter's configurations are considered to demonstrate the controllers' tracking capability; short-circuited (balanced fault) output, and grid-connected operation. This last case is evaluated operating as active filter and delivering fundamental component to a non-ideal grid.
\end{abstract}

\section{Introduction}

Current regulation in voltage source converters (VSC) for AC applications, such as motor drives, parallel active filters, wind turbines, static synchronous compensators and photovoltaic inverters, is a key and an underlying challenge. Several control strategies and implementation schemes can be found in the literature focused on different points of view; hysteresis control, internal model control (IMC), digital deadbeat, and rotating frame control (based in Park transforms) [1-8], among others. However, proportional-integral controllers (PI) and proportional-resonant controllers (PR) are shown as the most extended candidates.

When a synchronous reference frame (SRF) is applied, i.e. Park (dq0) transform, it is common to regulate the current using conventional PIs. Some recent efforts demonstrate that are a good option to be applied on voltage source converters connected to balanced and unbalanced grids [9-11]. The main drawback of this solution is the high computational burden when multiple frequencies and both positive and negative sequences need to be controlled, due to the mathematical transformations required to obtain null error at the desired frequencies [12].

A common alternative to the PI-SRF is the use of stationary reference frames, i.e. the natural reference $(a b c)$ or the Clarke transform $(\alpha b \gamma)$, where the so-called PR [13-15] can be employed. Whereas PI controllers present infinite gain at $0 \mathrm{~Hz}$, PR controllers have infinite gain at their resonant frequency $\omega_{0}$. A PI controller

$k_{p}+\frac{k_{i}}{s}$

implemented in a positive-sequence synchronous frame is not equivalent to a PR controller in stationary frame

$k_{p}+\frac{k_{i} s}{s^{2}+\omega_{0}^{2}}$

being $k_{p}$ and $k_{i}$ the controller's gain constants. In fact, it is the combination of a PI controller in a positive-sequence synchronous frame and another PI controller in a negative-sequence synchronous frame what makes it equivalent to a PR controller in stationary frame, both in

\footnotetext{
* Corresponding author.

E-mail address: daniel.heredero@citcea.upc.edu (D. Heredero-Peris).
} 


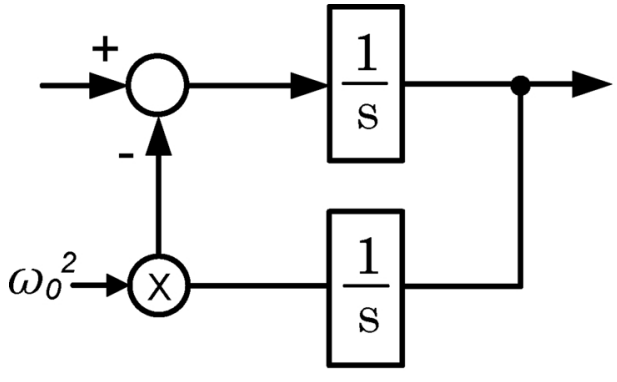

(a) SOGI scheme 1: $\frac{s}{s^{2}+\omega_{0}^{2}}$

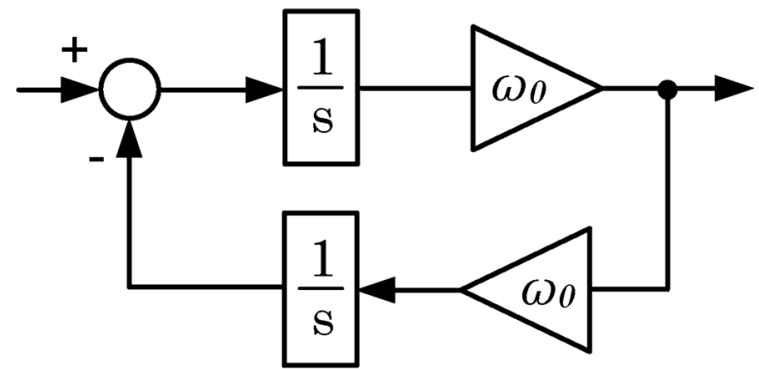

(b) SOGI scheme 2: $\frac{\omega_{0} s}{s^{2}+\omega_{0}^{2}}$

Fig. 1. Schemes concerning resonant controllers.

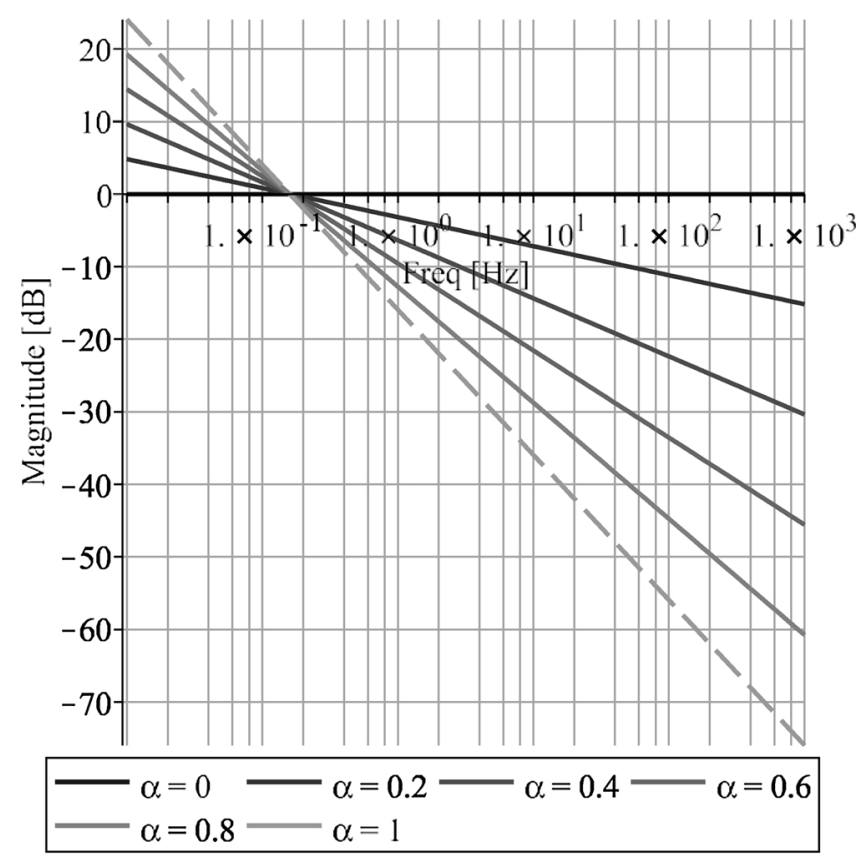

(a) Gain versus frequency

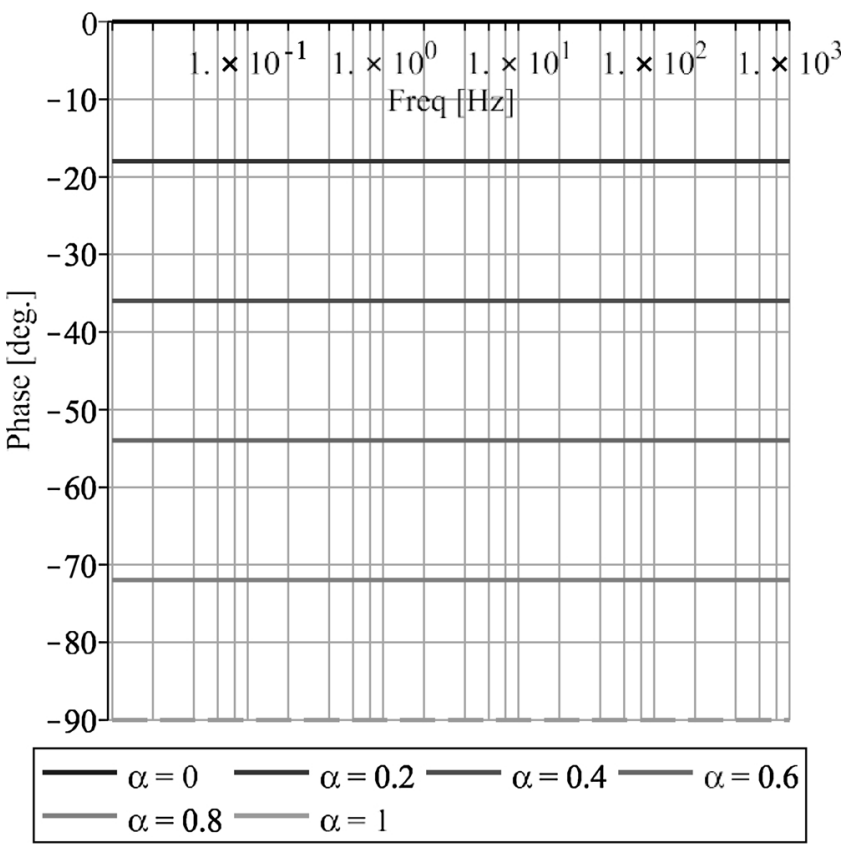

(b) Phase versus frequency

Fig. 2. Bode diagram of a fractal integrator $\frac{1}{s^{\alpha}}$, being $\alpha$ swept from 0 to 1 . steady-state and transient conditions $[16,17]$. Consequently, taking into account the feature of compensating for both positive and negative sequence components simultaneously, PR controller are able to deal with unbalanced three-phase systems $[14,18]$. PR controllers present narrow bandwidths around the resonant frequency. Some authors as in Refs. $[19,20]$ compare the performance obtained between the choice of selecting PI or PR controllers. Several alternatives are available regarding the discretization of the PR controller, which is specially sensitive to this process. This is extensively detailed in Ref. [21]. Related alternative control options can be found in the literature, such as sequence-decoupled resonant controllers [22] and complex-vector proportional-integral (CVPI) controllers [23,24]. Moreover, for large values of $\omega_{0}$, the delay caused by the sampling time and the modulation can affect the system performance and the stability. Adding a phase lead in the standard PR transfer function can compensate for the mentioned delay [25].

On the other side, the fractional order calculus (FOC) was first proposed in 1695 by Leibniz and L'Hôpital, when a half-order derivative was mentioned. The FOC is a generalization of integration and differentiation considering non-integer orders. Rational, irrational or even complex orders might be considered. This generalization allows for a more accurate description in the modelling of real systems. When the modelled system is analysed from a control point of view, four options can be combined: (i) integer-order plant and integer-order controller, (ii) integer-order plant and fractional-order controller, (iii) fractional-order plant and integer-order controller and (iv) fractionalorder for both plant and controller. In 1999 the fractional derivatives and integrators were used for the first time in the control field [26]. Podlubny proposed the fractional order $\mathrm{PI}^{\lambda} \mathrm{D}^{\mu}$ controller [27], being $\lambda$ and $\mu$ the non-integer order coefficients for the integrator and the derivative terms in the $s$ Laplace domain with respect to a conventional PID controller. Since then, $\mathrm{PI}^{\lambda} \mathrm{D}^{\mu}$ controllers have been used for control purposes [28-31]. Also, examples of fractional calculus applied to repetitive controllers can be found [32]. These controllers provide extra degrees of freedom that can improve the dynamic response.

To the best of the authors knowledge, the combination of the wellknown concepts of FOC and PR controllers has not been addressed in the literature until the authors proposed some preliminary results in Ref. [33]. The present paper analyses the potential benefits that lie behind this specific controller formulation in an exhaustive way, not only extending the analysis addressed in Ref. [33] but also proposing a tuning methodology, comparing different type of controllers and presenting more results (simulations and experiments). In this sense, the fractional proportional-resonant (FPR) controller proposal is presented in the continuous time domain and applied to the current control loop of a VSC. Essentially, this new controller contributes with one extra degree of freedom that allows an extension of the frequency bandwidth range, thus increasing the tracking capability of the system in steadystate and transiently in comparison with the conventional PR controller. This means that a certain range of frequencies can be effectively 


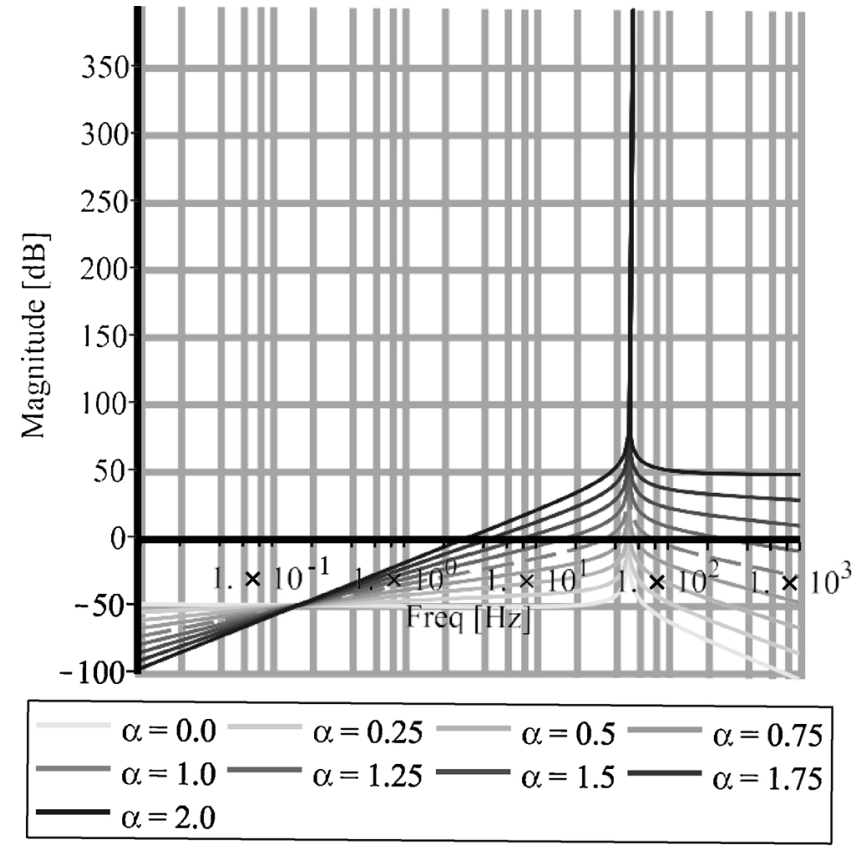

(a) Gain versus frequency

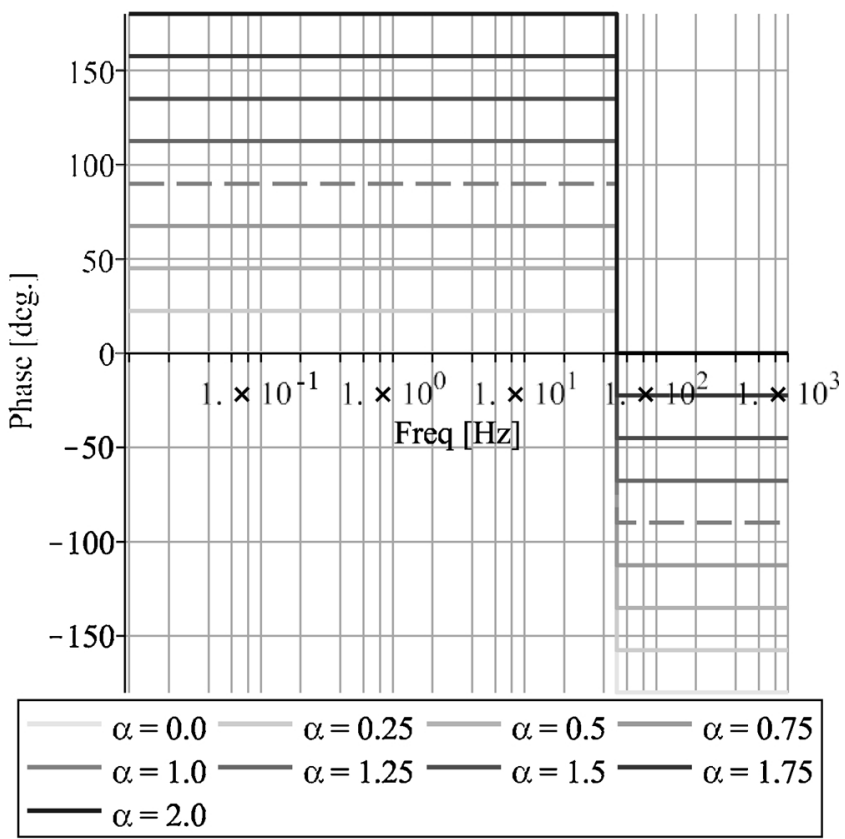

(b) Phase versus frequency

Fig. 3. Bode diagram of the FR $\frac{\omega_{0} s^{\alpha}}{s^{2}+\omega_{0}^{2}}$, being $\alpha$ swept from 0 to 2 .

controlled in steady-state with a single controller, not being necessary to include a resonant controller for each frequency (i.e. fundamental frequency and different harmonics) as in the PRHC case.

However, the new controller's formulation is not directly implementable due to the fact that the obtained transfer function is a nonrational polynomial in $s$ (Laplace variable). Thus, five different mathematical approaches are analysed and compared, suggesting Chareff's approximation as an adequate solution. Also, a tuning procedure is addressed in order to select suitable parameters for the controller. Finally, the controller is tested with simulations and a real laboratory test bench by means of a four-wire inverter under different conditions. Two different output configurations are used to prove the performance of the FPR controller; one is connecting the AC outputs terminals in

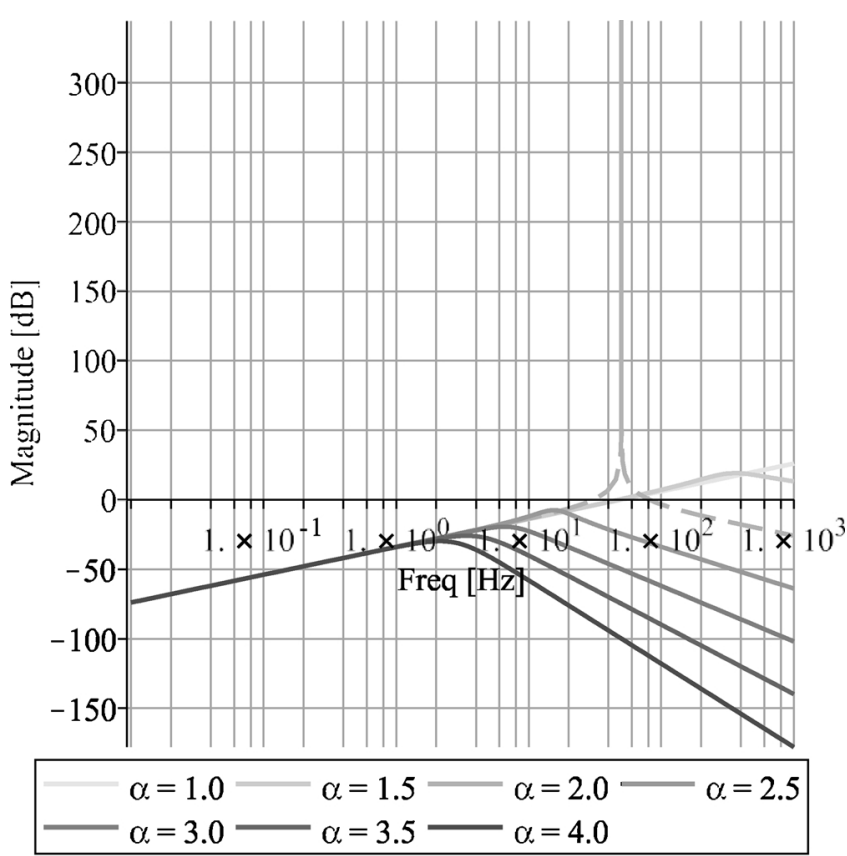

(a) Gain versus frequency

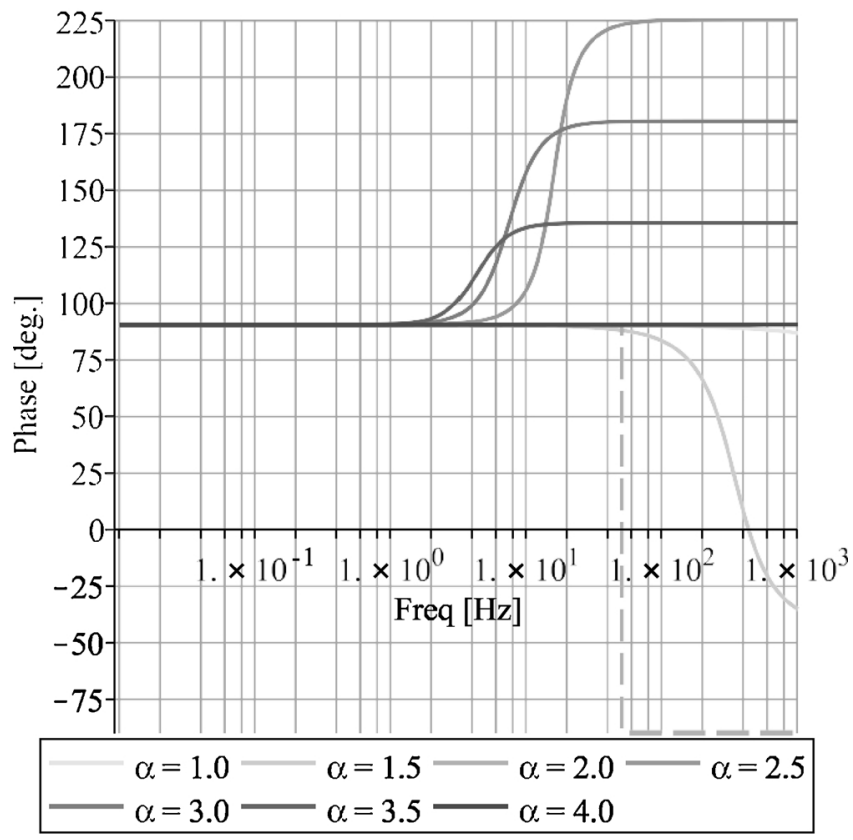

(b) Phase versus frequency

Fig. 4. Bode diagram of the FR $\frac{\omega_{0} s}{s^{\alpha}+\omega_{0}^{2}}$, being $\alpha$ swept from 1 to 4 .

short-circuit, and the other one is operating the inverter connected to the grid. This last scenario is in turn divided into two functionalities; one as an active filter, and the other one just delivering fundamental current but with a distorted grid. In this sense, balanced and unbalanced conditions are assumed for the performance evaluation. Some previous results of the enhancement obtained with the proposed FPR controlled where presented in Ref. [34] (European Union seventh framework programme FP7-ICT-2013-11-Smart Rural Grid-).

Bearing in mind the aforementioned aims, the paper is structured as follows. First, Section 2 introduces the FPR controller formulation. After, the tuning procedure and the implementation challenges are disclosed in Section 3. Then, a stability and sensitivity analysis of the new controller formulation is developed in Section 4. Experimental 


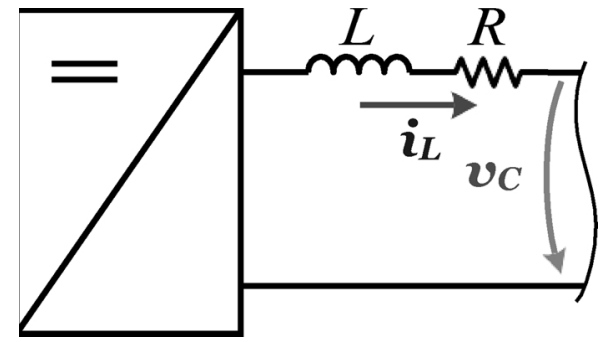

Fig. 5. VSC inverter with an L-type coupling filter.

implementation in a test platform and the results obtained are detailed in Section 5. Finally, the conclusions are summarized.

\section{The fractional resonant controller contribution}

This section presents the influence of non-integer order coefficients applied to stationary frame controllers. The conventional PR transfer function is illustrated and the result of adding the fractional exponents on different parts of the transfer function is compared.

\subsection{Conventional proportional-resonant controller formulation}

The transfer function of the conventional PR controller, also known as second order generalized integrator (SOGI), contains two poles located at $\pm j \omega_{0}$, being $\omega_{0}$ the resonant frequency, i.e. the controlled frequency with zero steady-state error. Fig. 1 illustrates two different implementations of the PR controller. An $\omega_{0}$ gain can be included in the numerator (Fig. 1(b)), which is useful for quadrature signal generation $[35,36]$. Hereinafter, the formulation used will be the one shown in Fig. 1(b). When multiple frequencies regulation is required, harmonic compensators (HC) are added to the main PR controller, obtaining the PRHC controller transfer function

$G_{\mathrm{PRHC}}(s)=\overbrace{k_{p}+k_{i} \frac{s \omega_{0}}{S^{2}+\omega_{0}^{2}}}^{P R}+\overbrace{\sum_{h=2}^{n} k_{i h} \frac{s \omega_{h}}{S^{2}+\omega_{h}^{2}}}^{H C}$

being $k_{p}, k_{i}$ and $k_{i h}$ the proportional and the integral gains of each resonant term, respectively, $h$ the harmonic component of the fundamental frequency $\omega_{0}$ and $n$ the highest harmonic component to be controlled. The $k_{i h}$ factor is obtained dividing $k_{i}$ by $h$ in order to compensate for the additional gain provided by $\omega_{h}$, thus scaling all integral gains equivalently [37]. Different tuning methods for the controller gains can be obtained by means of either time response [36] or frequency specifications $[38,17,39]$.

\subsection{The fractional resonant controller formulation}

The motivation behind using integro-differential operators is concerned with frequency domain analysis. The fractional integrator (FI) term $s^{-\alpha}(\alpha \in \mathbb{R})$ has infinite gain at $0 \mathrm{rad} / \mathrm{s}$ and less delay than a conventional integrator $s^{-1}$ at higher frequencies. If $\alpha \in[0,1]$ the phase shift provided by $s^{-\alpha}$ is $\alpha$ times lower than $s^{-1}$. The gain decreases proportionally as $\alpha$ is reduced from 1 to 0 . Fig. 2 illustrates this behaviour for different ideal FIs in the frequency domain. The key angular frequency in the gain diagram is $\omega=1 \mathrm{rad} / \mathrm{s}$ or $f=0.15915 \mathrm{~Hz}$. Therefore, if $\alpha$ increases, the gain is higher for angular frequencies below $1 \mathrm{rad} / \mathrm{s}$, and lower for frequencies above $1 \mathrm{rad} / \mathrm{s}$. The contrary happens if $\alpha$ decreases.

Now, if the $s^{\alpha}$ term is combined with the ordinary PR formulation (3), the obtained fractional resonant (FR) controller can be either
Table 1

Parameters used in the study.

\begin{tabular}{lll}
\hline Parameter & Value & Units \\
\hline$L_{b}$ & 500 & $\mu \mathrm{H}$ \\
$R_{b}$ & 50 & $\mathrm{~m} \Omega$ \\
Settling angular frequency $\left(\omega_{0}\right)$ & $100 \pi$ & $\mathrm{rad} / \mathrm{s}$ \\
Settling time $\left(t_{s}\right)$ & 2.5 & $\mathrm{~ms}$ \\
Damping factor $(\xi)$ & 0.95 & \\
\hline
\end{tabular}

described by (4) or (5)

$G_{F R}(s)=\frac{\omega_{0} s^{\alpha}}{s^{2}+\omega_{0}^{2}}$

$G_{F R}(s)=\frac{\omega_{0} s}{s^{\alpha}+\omega_{0}^{2}}$

On one hand, when (4) is considered, if the fractional exponent is swept from $\alpha \in[0,2]$, it can be seen that the infinite gain is maintained at $\omega_{0}$ (Fig. 3) because the denominator remains unchanged. However, the fact of changing the numerator brings more gain near $\omega_{0}$ when $\alpha \in$ $(1,2]$, as depicted in Fig. 3(a). Furthermore, the gain shift for higher frequencies (Fig. 3(b)), shows a behaviour similar to the FI depicted in Fig. 2(b). When $\alpha \in[0,1)$, poorer tracking capabilities are expected in comparison with the $\alpha=1$ case. For $\alpha>2$, the phase shift at $\omega_{0}$ changes from lagging to leading. Due to this reason and in order to avoid stability issues, $\alpha>2$ case is excluded from the scope of this work. Moreover, it should be noted that the key factor for tracking a signal properly is that the phase delay has to be as small as possible. In this sense, (4) becomes an interesting alternative to PRHC formulation.

On the other hand, considering (5), with $\alpha \in[1,4]$ it can be observed that the infinite gain is lost and displaced (Fig. 4). Thereupon, (5) is rejected as a suitable controller for tracking sinusoidal waveforms.

\section{Tuning method and implementation of fractional proportional- resonant controllers}

This section focuses on the current control loop of a VSC using the controller based on (4). Accordingly, Fig. 5 shows a conceptual scheme of a VSC with an L-type coupling filter connected to an external AC voltage source or a load. From now on, this will be the system considered for the analysis, being $i_{L}$ the regulated current.

First of all, the new fractional proportional-resonant (FPR) controller is presented. Then, the tuning procedure is introduced and different variations over the hardware and software parameters are analysed in the frequency domain through Bode diagrams. Note that the present proposal based on (4) is not directly implementable in the Laplace $s$-domain, but it can be analysed mathematically computing the gain and phase in the frequency domain.

\subsection{The fractional proportional-resonant controller}

According to the aforementioned disclosures in Section 2.2, the combination of (4) with a proportional controller results in the FPR controller

$F P R(s)=k_{p}+k_{i} \frac{\omega_{0} s^{\alpha}}{s^{2}+\omega_{0}^{2}}$

being $k_{p}$ and $k_{i}$ the proportional and integral gains. 


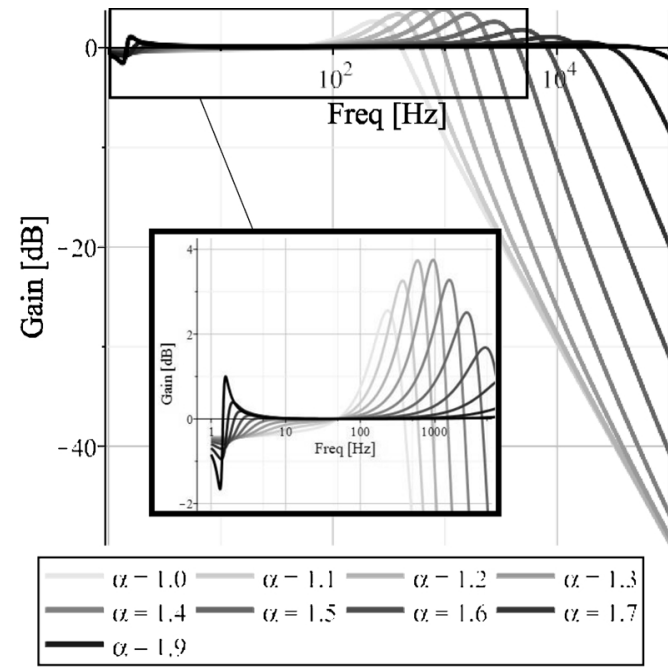

(a) Gain versus frequency

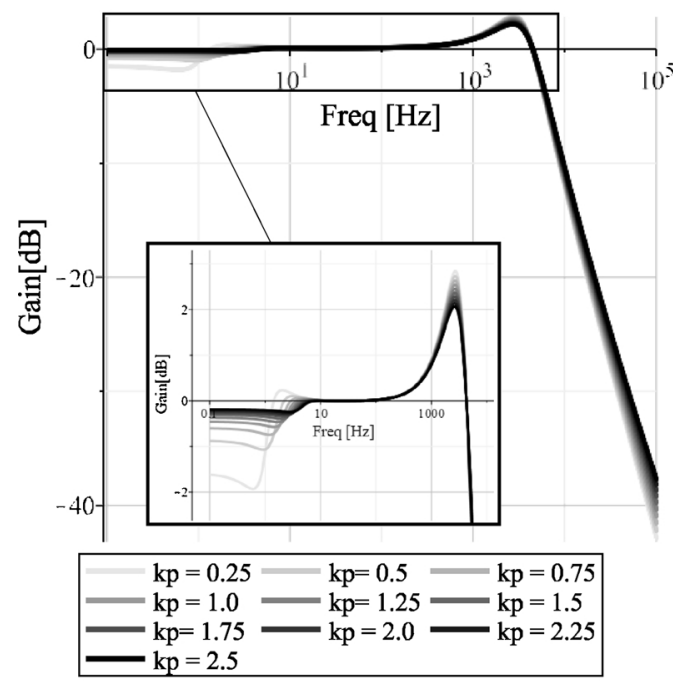

(c) Gain versus frequency

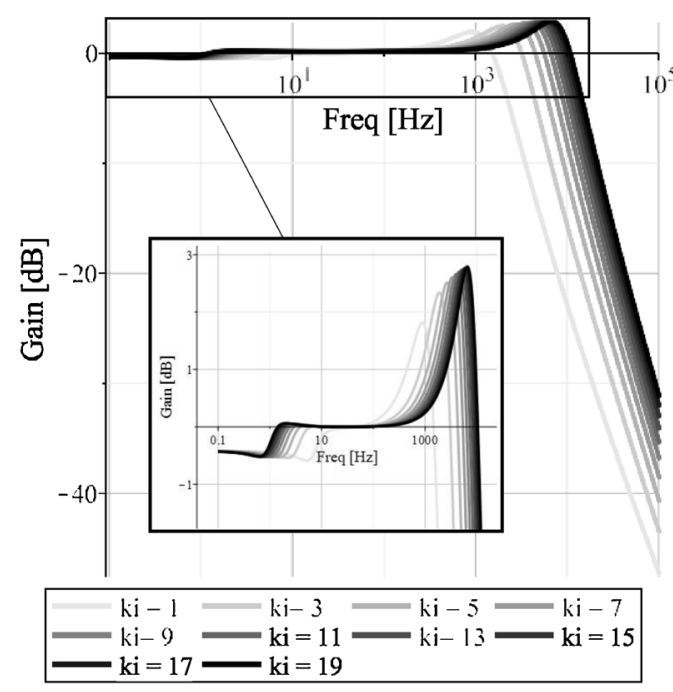

(e) Gain versus frequency

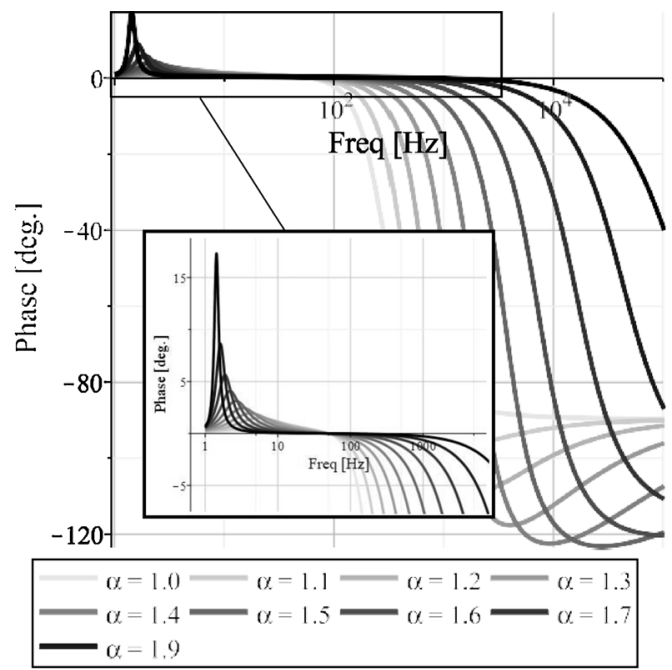

(b) Phase versus frequency

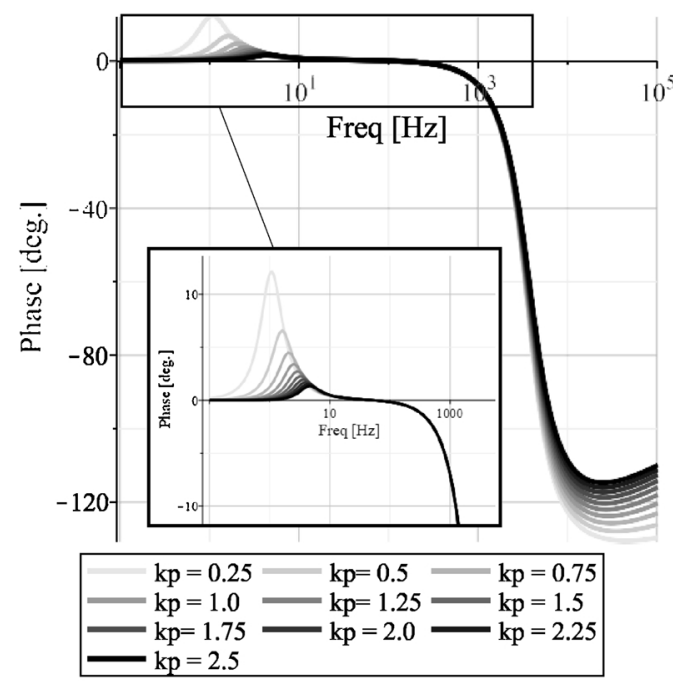

(d) Phase versus frequency

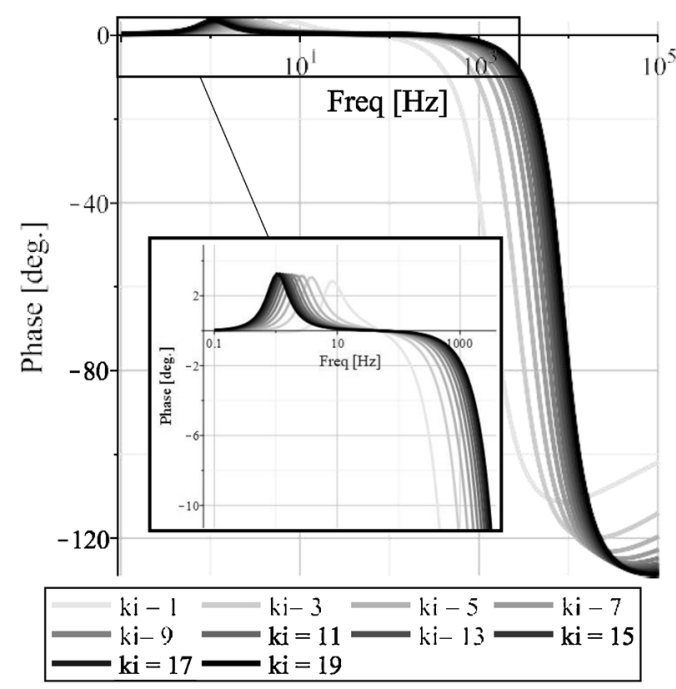

(f) Phase versus frequency

Fig. 6. Closed loop Bode diagram when the FPR parameters are swept. 


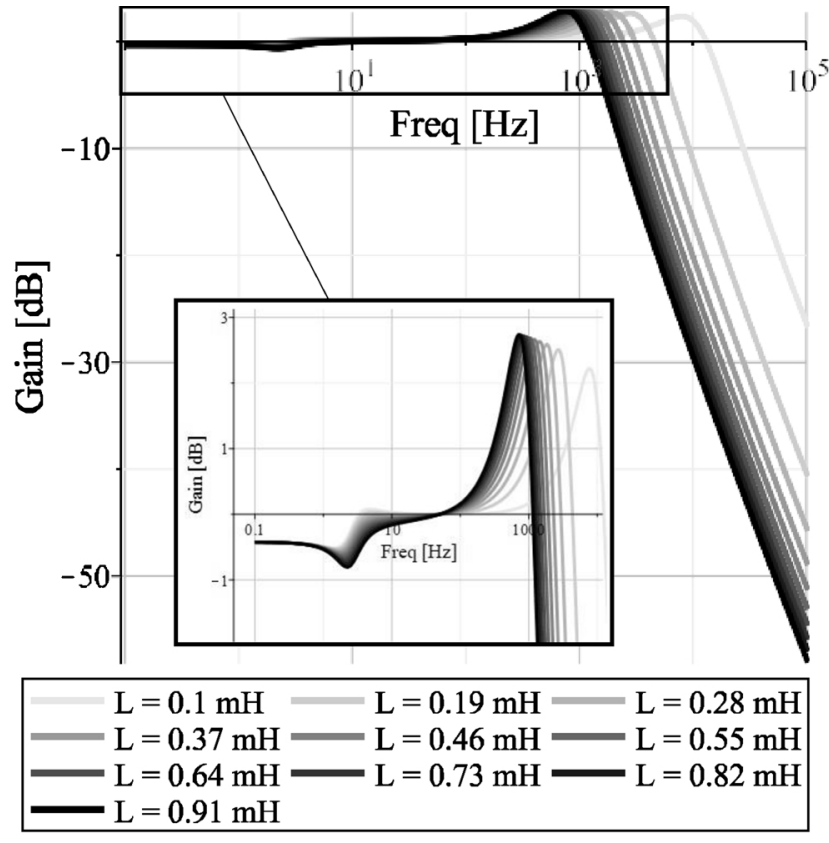

(a) Gain versus frequency

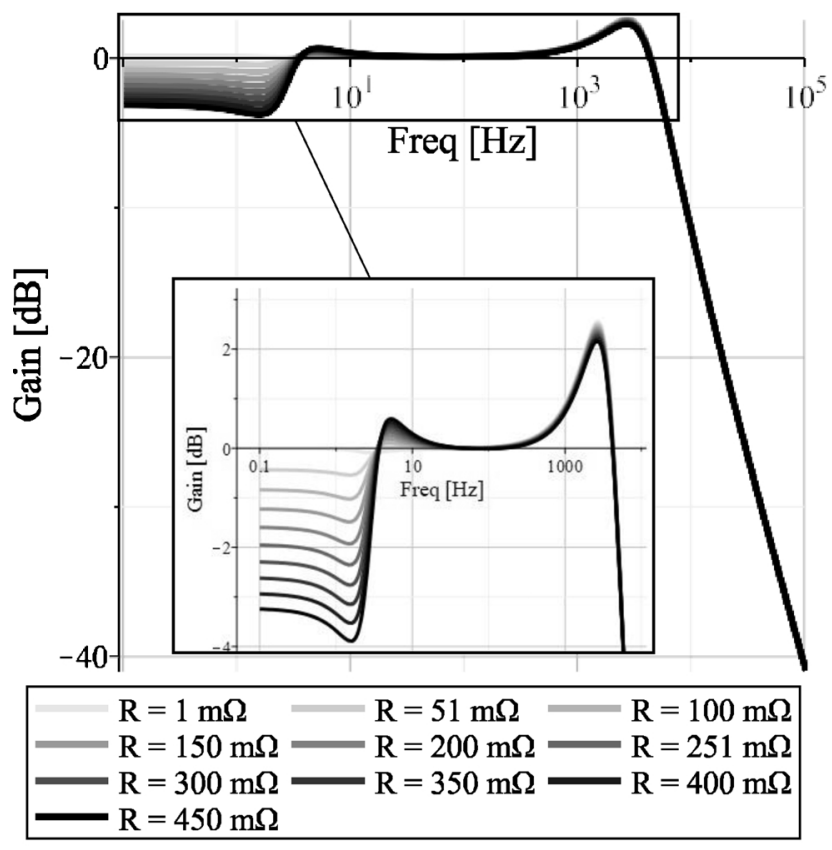

(c) Gain versus frequency

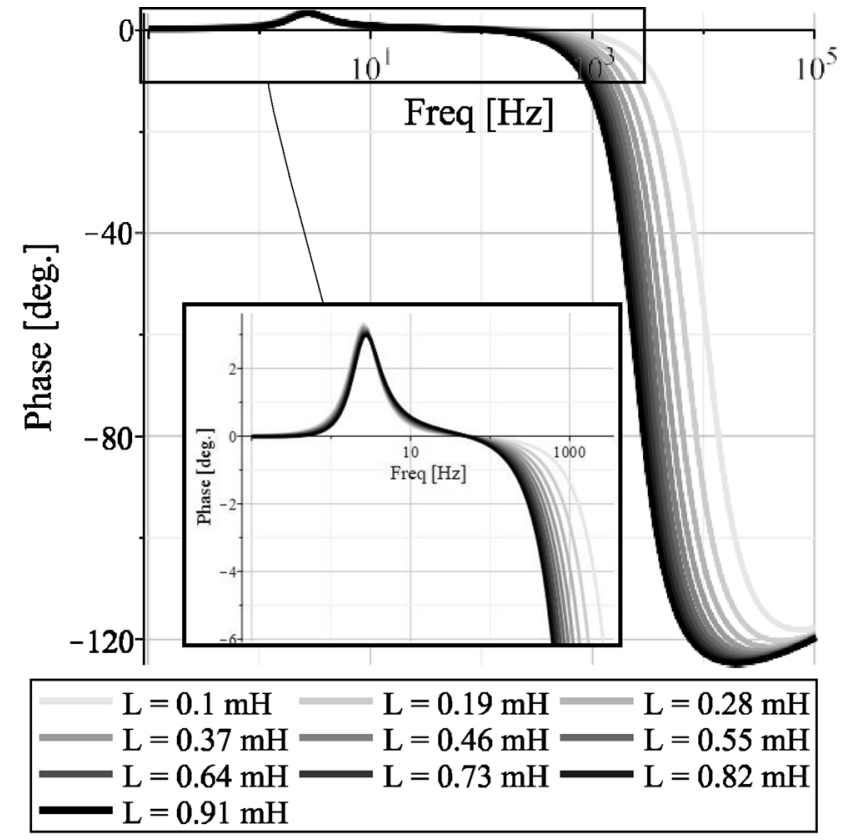

(b) Phase versus frequency

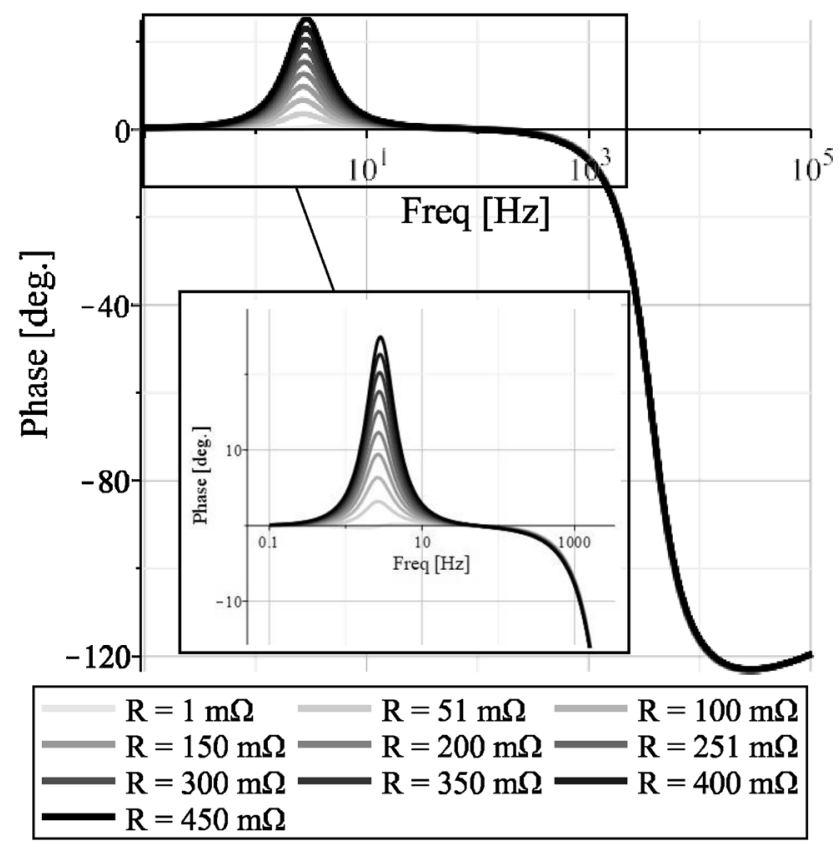

(d) Phase versus frequency

Fig. 7. Closed loop Bode diagram when the VSC inverter parameters are swept.

\subsection{Frequency response closed loop analysis}

The closed loop (CL) transfer function of the system consisting of the FPR controller and the plant, assuming a feed-forward structure to compensate for $v_{c}$, is

$G_{C L}(s)=\frac{I_{L}(s)}{I_{L}^{*}(s)}=\frac{\frac{1}{L s+R}\left(k_{p}+k_{i} \frac{\omega_{0} s^{\alpha}}{s^{2}+\omega_{0}^{2}}\right)}{1+\frac{1}{L s+R}\left(k_{p}+k_{i} \frac{\omega_{0} s^{\alpha}}{s^{2}+\omega_{0}^{2}}\right)}$

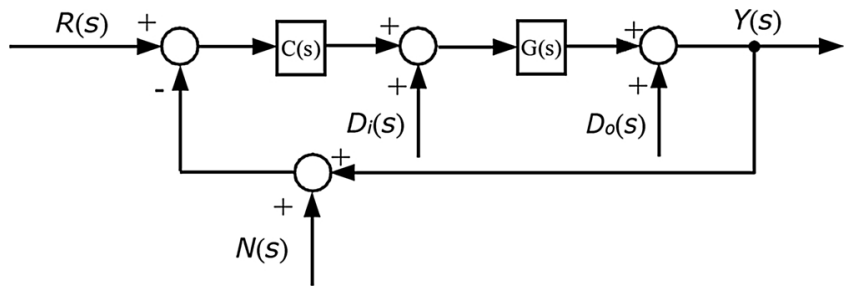

Fig. 8. Sensitivity control scheme. 


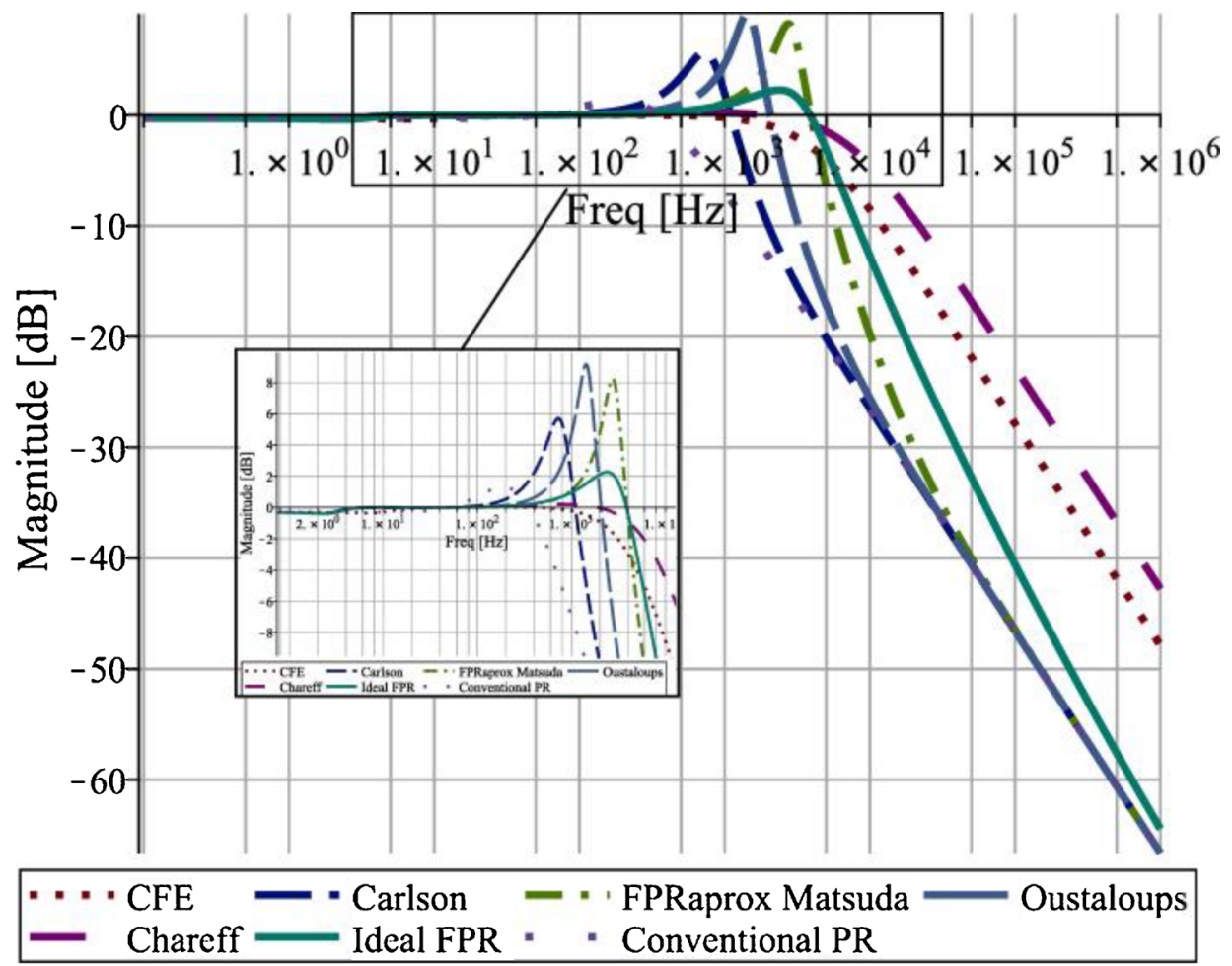

(a) Gain versus frequency

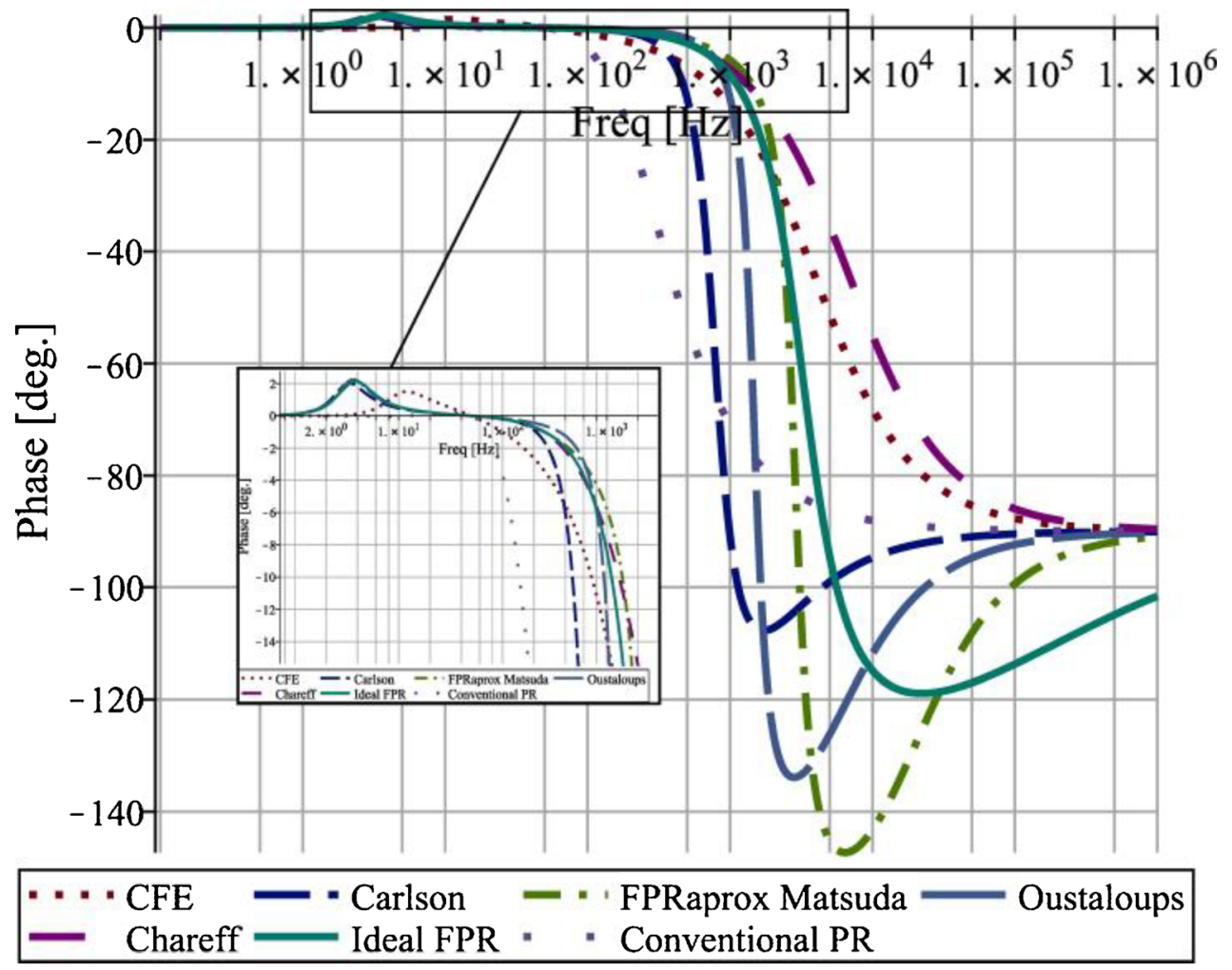

(b) Phase versus frequency

Fig. 9. Bode diagram comparison when different continuous time approximations of $s^{\alpha}$ are applied. 
Table 2

Summary of the used continuous time fractal approximation parameters.

\begin{tabular}{llll}
\hline Approximation method & Parameter & Value & Units \\
\hline \multirow{2}{*}{ CFE } & Order & 5 & - \\
\multirow{3}{*}{ Carlson } & $\mathrm{T}$ & 0.05 & - \\
Matsuda & $i$ & 2 & - \\
& $k$ & 8 & - \\
Oustaloup & $\omega_{k}$ & $1-1000$ & $\mathrm{rad} / \mathrm{s}$ \\
& $\omega_{b}$ & 0.001 & $\mathrm{rad} / \mathrm{s}$ \\
& $\omega_{h}$ & 1000 & $\mathrm{rad} / \mathrm{s}$ \\
Chareff & $\mathrm{N}$ & 2 & - \\
& $p_{T}$ & 1 & - \\
& $\mathrm{y}$ & 2 & $\mathrm{~dB}$ \\
& $\mathrm{n}$ & 4 & - \\
\hline
\end{tabular}

The output inductance $L$ also contains an equivalent series resistance $R$. The superscript * indicates set-point. Regarding the FPR controller tuning procedure, three parameters can be considered: (i) $k_{p}$ gain, (ii) $k_{i}$ gain and (iii) non-integer exponent $\alpha$. This last parameter provides an additional degree of freedom that allows for one extra condition to be imposed to the system dynamics response.

First of all, the effect of the hardware and software parameters on the CL frequency response is studied. The values used are summarized in Table 1. Thereupon, according to the time response tuning procedure presented in Ref. [36] for conventional discrete-time PR controllers, $k_{p}=1.44$ and $k_{i}=4.28$ are obtained, with a sampling frequency of $30 \mathrm{kHz}$. As the objective of this section is to use some $k_{p}$ and $k_{i}$ gains as pattern values, the base values will be $k_{p b}=1$ and $k_{i b}=5$.

Fig. 6 depicts the CL Bode diagrams of (7) when the controller parameters are modified:

- $\alpha$ variation. It can be seen in Fig. 6(a) and (b) that the frequency behaviour is improved over conventional PR controllers $\left(\alpha_{b}=1\right)$. The higher the $\alpha$ value, the wider the controllable bandwidth and the lower the delay obtained. Moreover, the resonance peak at low frequencies $(\simeq 10 \mathrm{rad} / \mathrm{s}$ or $1.5915 \mathrm{~Hz})$ and the bandwidth are increased for high values of $\alpha$. Also, a lead phase peak appears at low frequencies.

- $k_{p}$ variation. Fig. 6(c) and (d) show an almost unaltered frequency behaviour. However, if the $k_{p}$ increases a displacement to lower frequencies and a gain magnification of the peak in the phase plot can be observed.

- $k_{i}$ variation. Fig. 6(e) and (f) show that higher $k_{i}$ gains lead to a displacement of the gain excitation peak to the right side, whereas the lead phase region is displaced to the left. However, note that the differences are minor.

On the other hand, Fig. 7 depicts the CL Bode diagrams of (7) when the plant parameters are modified. This analysis permits to examine the effect on the frequency steady-state response when an error on the system parametrization is made or a variation of the parameters occurs during operation. Thus, if the system parameters are modified:

- $L$ variation. Fig. 7(a) and (b) illustrates that when $L$ is increased, with the rest of parameters kept constant, the frequency behaviour worsens. The higher the error above $L_{b}$, the lower the steady-state features. It should be remarked that the low frequency region (below $100 \pi \mathrm{rad} / \mathrm{s}$ or $50 \mathrm{~Hz}$ ) is almost unaffected.
- $R$ variation. Fig. 7(c) and (d) shows the frequency response when the equivalent series resistance is modified. Note that this case presents opposite results with respect to the ones presented in the $L$ variation analysis. In this case, the high frequency region (above $100 \pi \mathrm{rad} / \mathrm{s}$ or $50 \mathrm{~Hz}$ ) is almost unaffected.

\subsection{Stability effect of $\alpha$}

A stability analysis of current and voltage resonant controllers for VSC is derived in Ref. [37]. In the particular case of current control loop, it is demonstrated that in continuous time the system is always stable, independently of the plant parameters (inductance or parasitic resistance) or of the PR parameters (proportional or integral gain). However, this results are addressed only for integer $s$ variables. In this section, the same analysis from Ref. [37] is done, extending it for the FPR formulation shown in (6). The open loop transfer function is

$O_{L_{i}}(s)=\frac{k_{p i}\left(s^{2}+\omega_{0}^{2}\right)+k_{i i} \omega_{0} s^{\alpha}}{\left(s^{2}+\omega_{0}^{2}\right)(L s+R)}$

The significant frequency values of the Nyquist plot are:

- $\omega=0$ (Start point). $\mathfrak{R}\left(O L_{i}(j \omega)\right)=\frac{k_{P_{i}}}{R}$ and $\mathfrak{J}\left(O L_{i}(j \omega)\right)=0$.

- $\omega=\omega_{0} \quad$ (Resonance). $\quad \lim _{\omega \rightarrow \omega_{0}} \mp\left\{\mathfrak{R}\left(O L_{i}(j \omega)\right)\right\}= \pm \infty \quad$ and $\lim _{\omega \rightarrow \omega_{0}} \mp\left\{\mathfrak{J}\left(O L_{i}(j \omega)\right)\right\}= \pm \infty$, following an asymptote with $\frac{R \sin \left(\frac{\alpha \pi}{2}\right)-\omega L \cos \left(\frac{\alpha \pi}{2}\right)}{R \cos \left(\frac{\alpha \pi}{2}\right)+\omega L \sin \left(\frac{\alpha \pi}{2}\right)}$ slope. An infinite clockwise encirclement takes place at this frequency because of the $\pi$ phase lag inherent to the resonant term.

- $\omega \rightarrow+\infty \quad$ (End point). $\quad \lim _{\omega \rightarrow+\infty}\left\{\mathfrak{R}\left(O L_{i}(j \omega)\right)\right\}=0 \quad$ and $\lim _{\omega \rightarrow+\infty}\left\{\mathfrak{J}\left(O L_{i}(j \omega)\right)\right\}=0$.

\subsection{The tuning procedure}

According to the results shown in Section 3.2, it can be deduced that the proper selection of an $\alpha$ value can be used to improve the control tracking response of an alternating signal. Hence, the following tuning procedure is suggested:

1. Tune a conventional PR controller (without HC) either with time domain or frequency specifications, such as the ones presented in Refs. $[36,38]$. Then, the base $k_{p}$ and $k_{i}$ gains are obtained.

2. Depict the different Bode plots considering $\alpha$ from 1 to 2 using the $k_{p}$ and $k_{i}$ gains obtained in step 1 .

3. Select one $\alpha$ value according to a desired delay at a higher frequency above $\omega_{0}$.

4. Examine a proper approximation to the $s^{\alpha-1}$ term that makes implementable the non-integer part of (6).

5. Check whether the selected $\alpha$ presents robust behaviour if the system parameters ( $L$ and $R$ ) are improperly modelled. Different alternatives can be considered. In this case, the use of zero pole maps and root locus will be considered. It is also recommended to consider a sensitivity analysis. The sensitivity transfer functions that can be obtained (see Fig. 8), are described by

$S(s)=\frac{1}{1+C(s) G(s)}$

$T(s)=\frac{C(s) G(s)}{1+C(s) G(s)}$ 


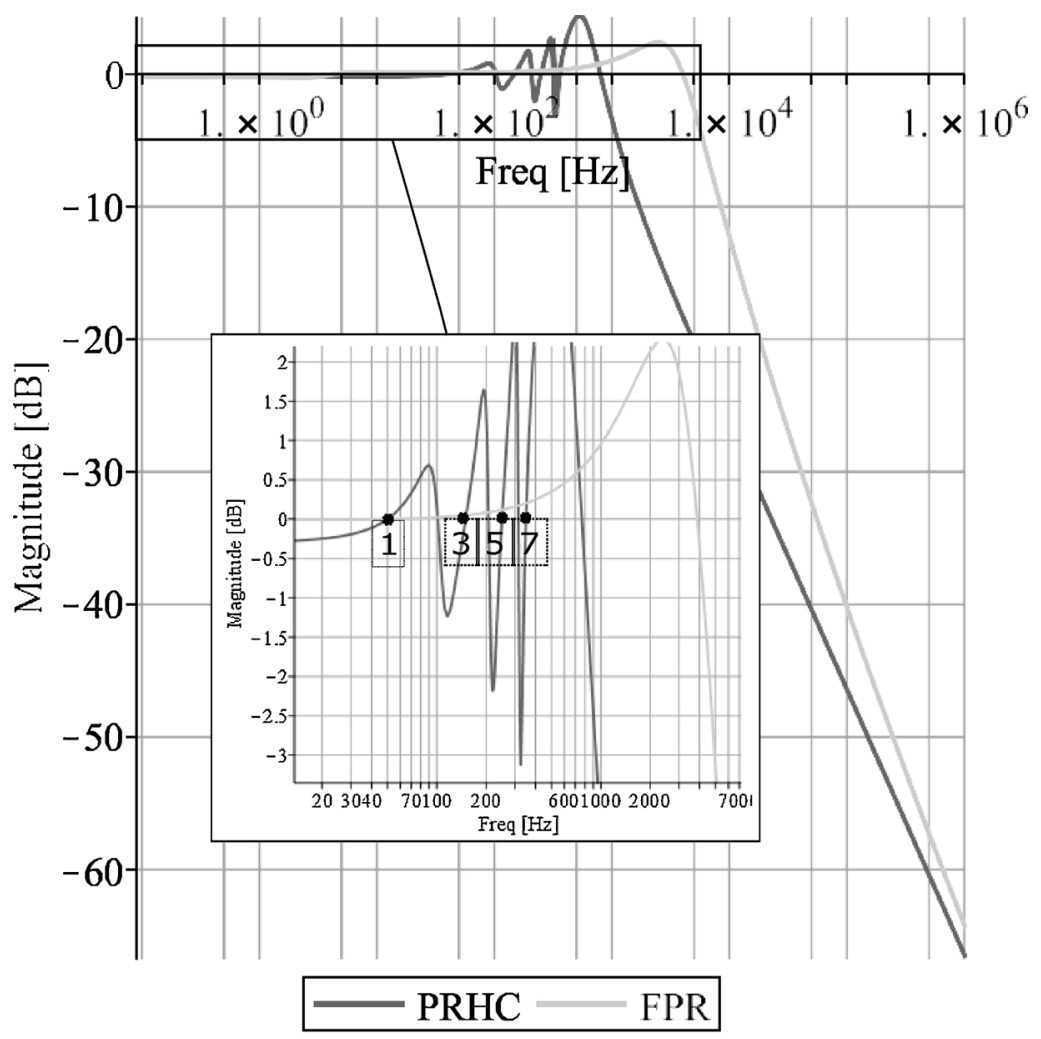

(a) Gain versus frequency

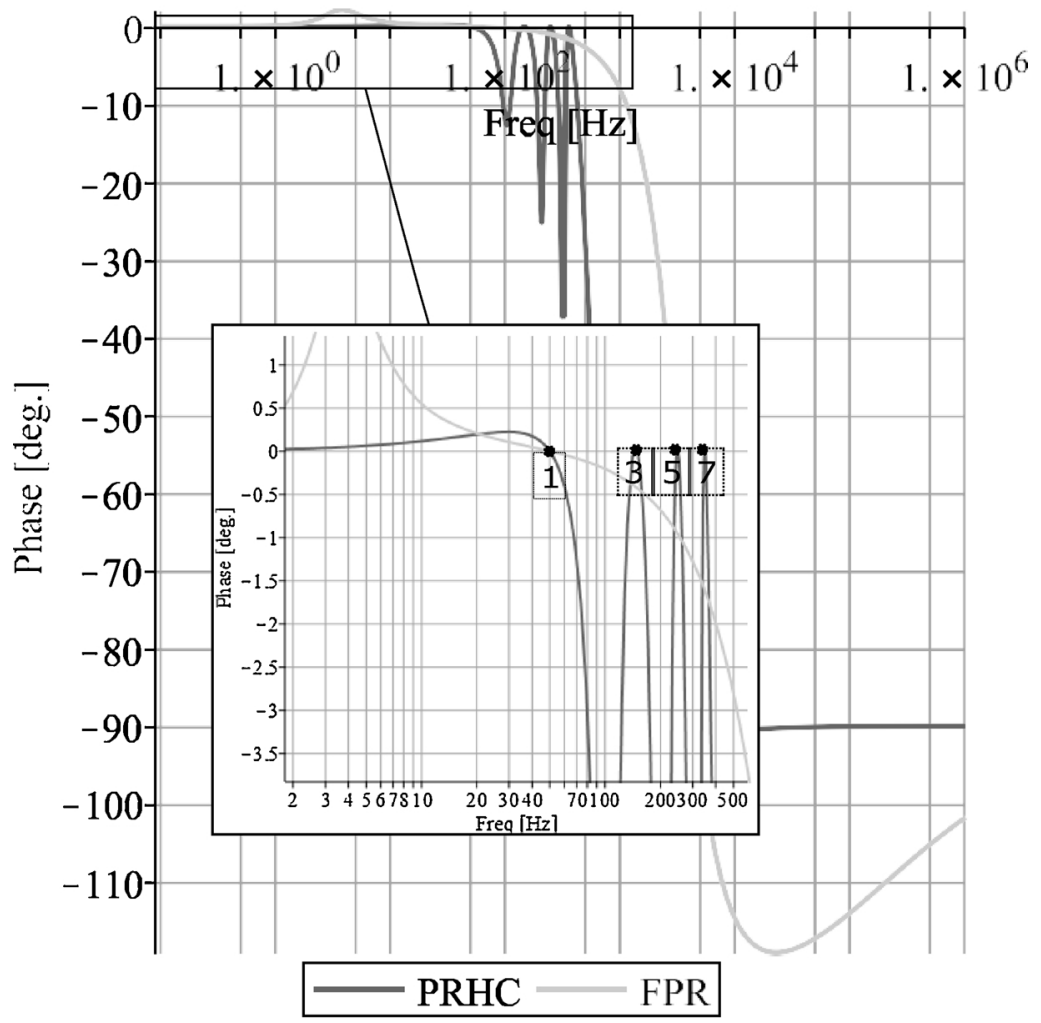

(b) Phase versus frequency

Fig. 10. Bode diagram comparing the PRHC controller (3th, 5th and 7th compensators) and the proposed FPR controller with $\alpha=1.5$. 


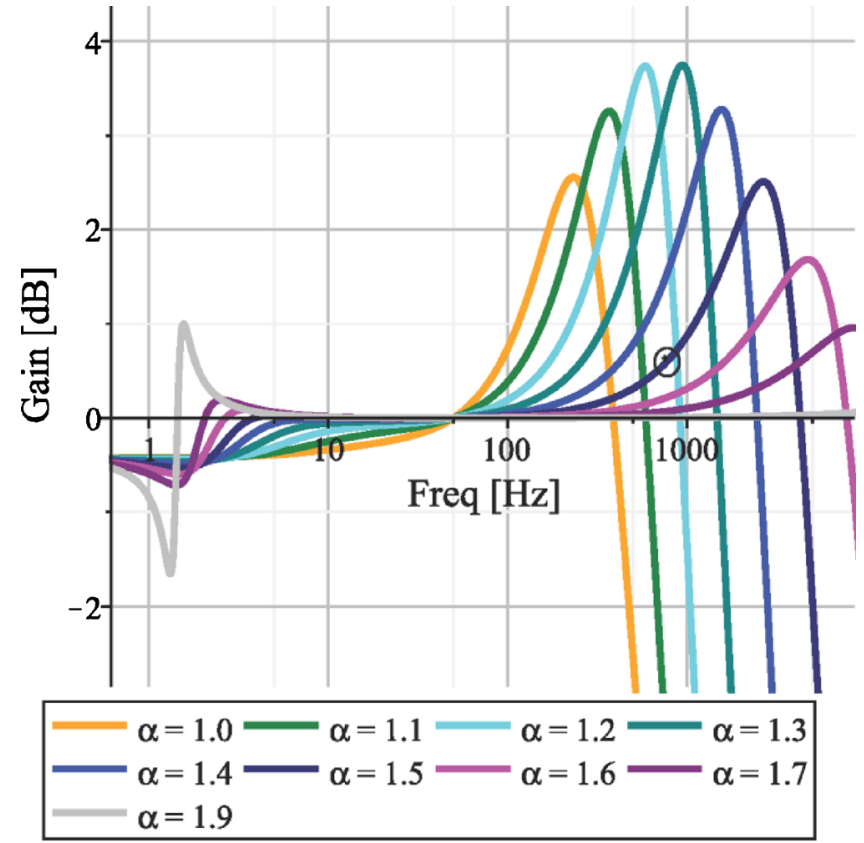

(a) Gain versus frequency

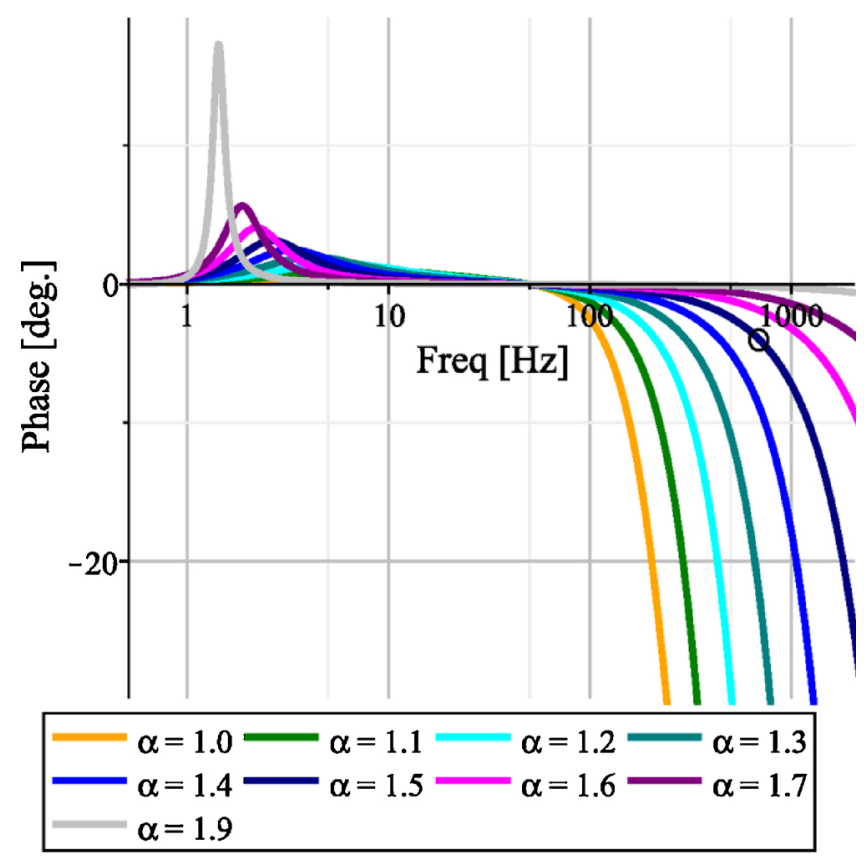

(b) Phase versus frequency

Fig. 11. Closed loop Bode diagram zoom when the $\alpha$ are swept in (7).

$S_{i}(s)=\frac{G(s)}{1+C(s) G(s)}$

$S_{u}(s)=\frac{C(s)}{1+C(s) G(s)}$

being $S(s)$ the conventional sensitivity, $T(s)$ the complementary sensitivity (CL transfer function), $S_{i}(s)$ the input sensitivity and $S_{u}(s)$ the control sensitivity.

6. Check the correct tracking response on simulations.

Note that a fundamental aspect while tracking an electrical signal is to eliminate the phase delay when several harmonic components have to be regulated. It is also possible to select a new $\alpha$ in step 3 to set the gain at a particular high frequency but, as it has been mentioned, it is more effective to modify the frequency response in terms of delay.

\subsection{Implementation}

As mentioned before (step 4 of Section 3.4) the implementation of the FPR controller is not directly feasible due to the non-rational behaviour of the transfer function. For this reason, it is necessary to apply an implementable form of the controller by means of an approximation with integer exponents. Several references [40-42] propose different approximations of the term $s^{\alpha}$. The following list summarizes the most relevant ones:

- Continued fraction expansion (CFE). For $\omega T>>1$, the expansion of (10) is considered, whereas for $\omega<<1$, the equation that has to be expanded is (11), being $\omega$ the angular frequency and $T$ a scale factor.

$G_{h}(s)=\frac{1}{(1+s T)^{\alpha}}$

$G_{l}(s)=\left(1+\frac{1}{s}\right)^{\alpha}$

- Carlson's approximation. Carlson proposed a method derived from Newton's approximation to obtain an iterative formula for the $\alpha$-root. The method begins with the following condition:

$H(s)^{1 / \alpha}-G(s)=0$

Defining $\alpha=1 / q, m=q / 2$, and considering $H_{0}(s)=1$, the approximation for the $i$-th iteration is defined by

$H_{i}(s)=H_{i-1}(s) \frac{(q-m)\left(H_{i-1}(s)\right)^{2}+(q+m) G(s)}{(q+m)\left(H_{i-1}(s)\right)^{2}+(q-m) G(s)}$

- Matsuda's approximation. It is a CFE with function values known at some frequencies $\omega_{k}(k=0,1,2, \ldots, n)$ :

$H(s)=a_{0}+\frac{s-\omega_{0}}{a_{1}+} \frac{s-\omega_{1}}{a_{2}+} \frac{s-\omega_{2}}{a_{3}+} \cdots \frac{s-\omega_{n}}{a_{n+1}+}$

$a_{i}=\nu_{i}\left(\omega_{i}\right)$

$\nu_{0}(s)=H(s)$

$v_{i+1}(s)=\frac{s-\omega_{i}}{v_{i}(\mathrm{~s})-a_{i}}$

The frequencies are obtained by means of a logarithmic spaced set.

- Oustaloup's approximation. This approach is calculated as a rational function considering $H(s)=s^{\delta}\left(\delta \in \mathbb{R}^{+}\right)$:

$H(s) \cong C^{\delta} \prod_{k=-N}^{N} \frac{1+s / \omega_{k}^{\prime}}{1+s / \omega_{k}}$

being $2 N+1$ the number of total poles or zeros. It is possible to compute the recursive formulation using the following set of intermediate relations:

$C=\frac{\omega_{u}}{\omega_{h}}=\frac{\omega_{l}}{\omega_{u}}$ 


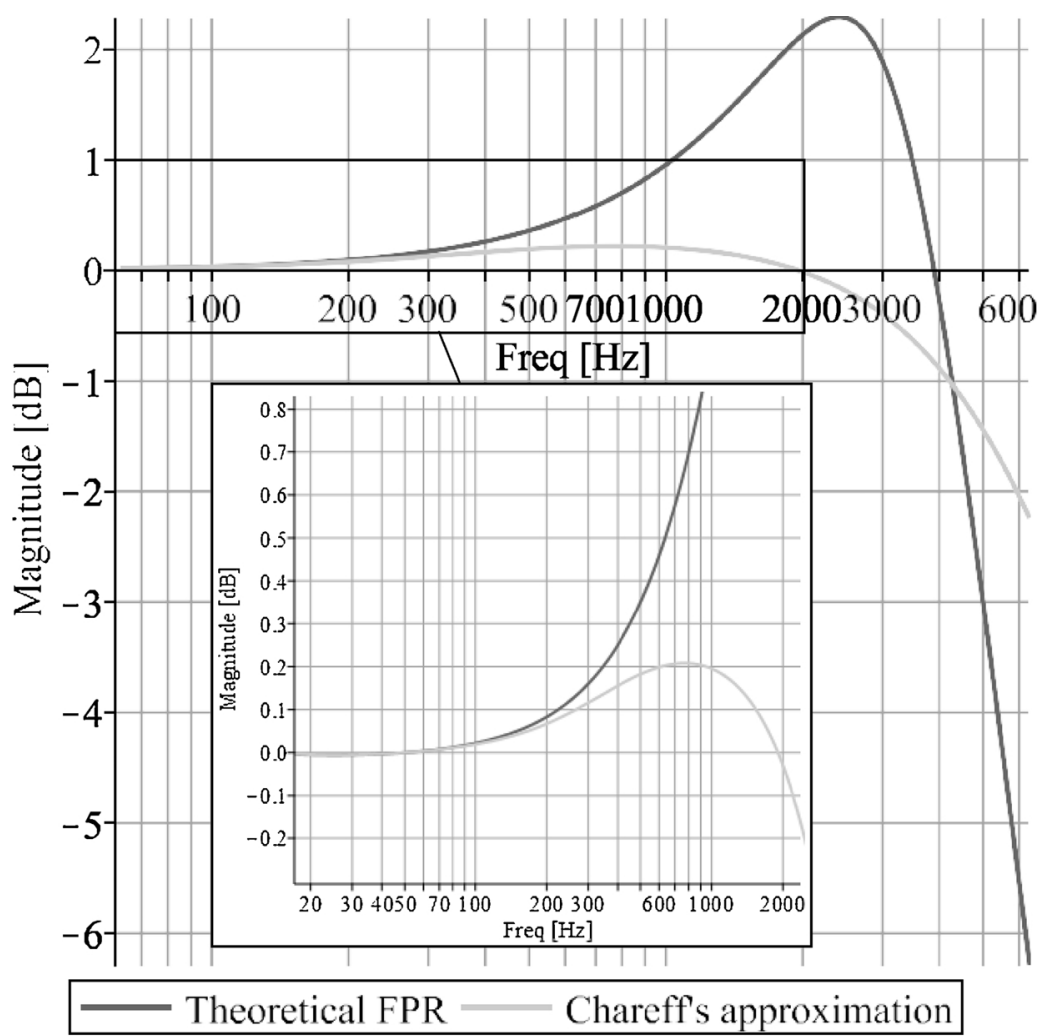

(a) Gain versus frequency

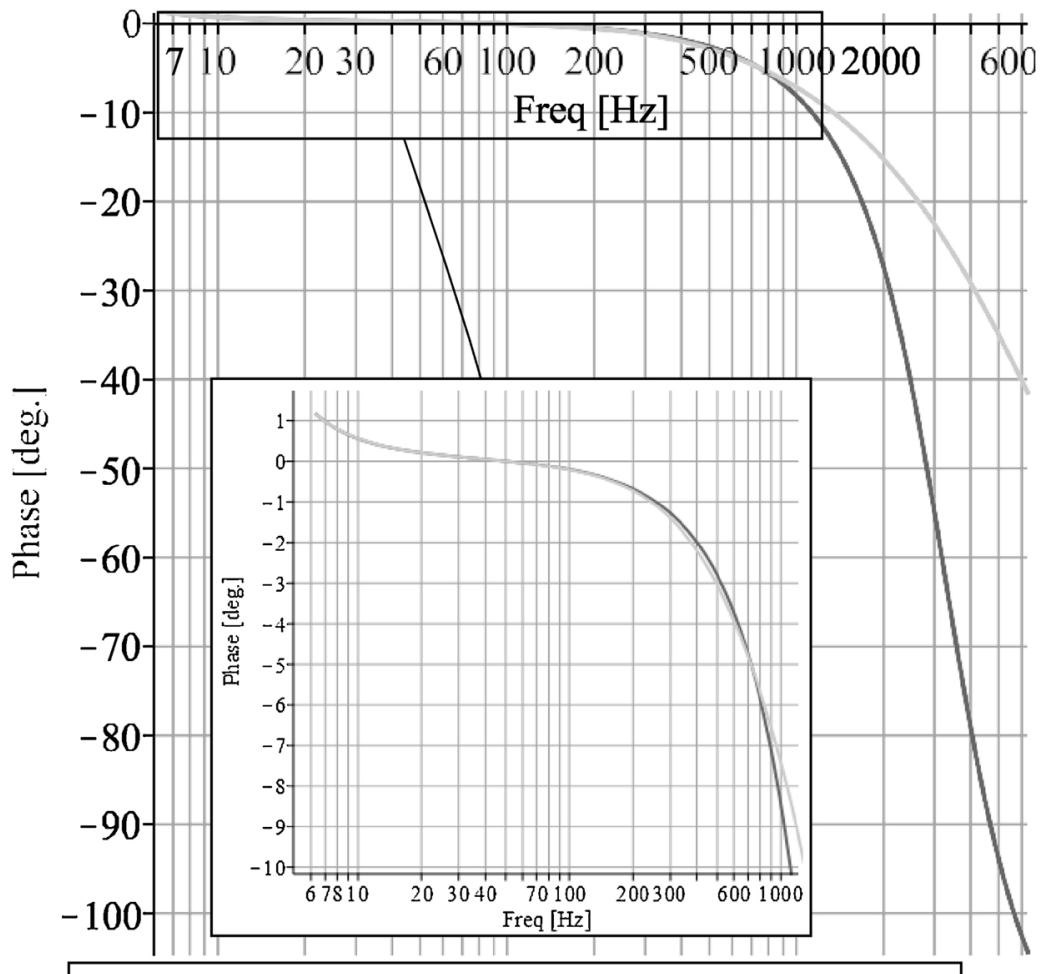

Theoretical FPR — Chareff's approximation

(b) Phase versus frequency

Fig. 12. Approximation comparison for the term $s^{\alpha-1}$ in the CL. 


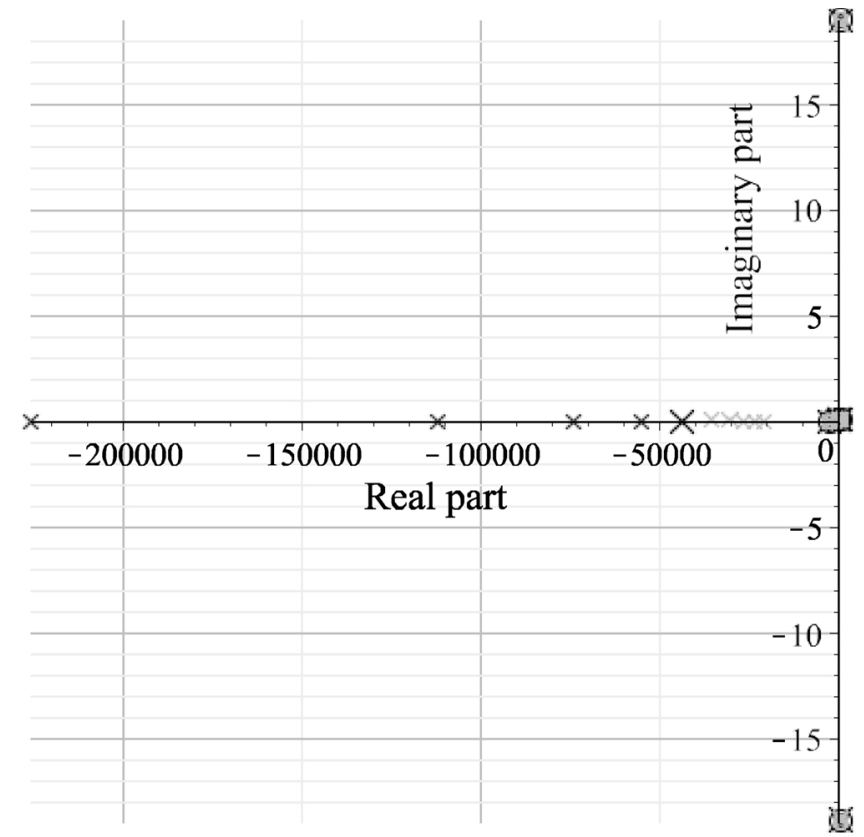

(a) Robustness analysis when $L$ is considered

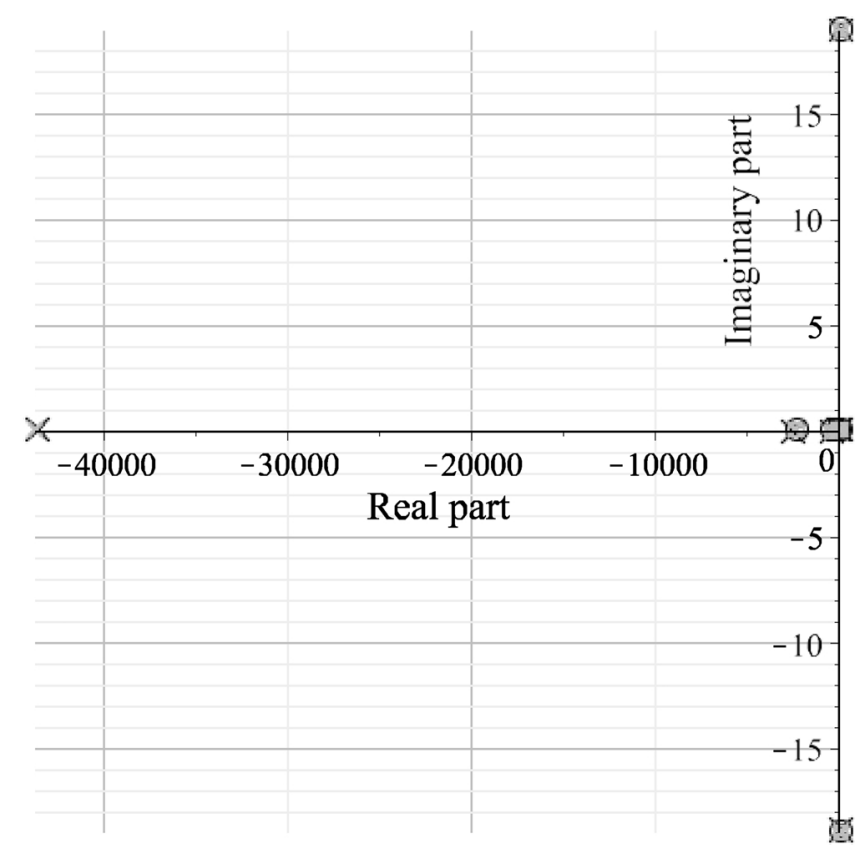

(b) Robustness analysis when $R$ is considered

Fig. 13. Zero-pole map analysis. Base values are multiplied by a scale factor from 0.2 to 2 in steps of 0.2 .

$\omega_{k}=\omega_{l}\left(\frac{\omega_{h}}{\omega_{l}}\right)^{\frac{k+N+\frac{(1+\delta)}{2}}{2 N+1}}$

$\omega_{k}^{\prime}=\omega_{l}\left(\frac{\omega_{h}}{\omega_{l}}\right)^{\frac{k+N+\frac{(1-\delta)}{2}}{2 N+1}}$ with $\omega_{u}=\sqrt{\omega_{h} \omega_{l}}$ being the unit gain frequency and the frequency band midpoint of a band of frequencies geometrically distributed around it.

- Chareff's approximation. If the desired transfer function has the form of

$H(s)=\frac{1}{\left(1+\frac{s}{p_{T}}\right)^{\alpha}}$

the proposed approach is based on the factorization

$H(s) \cong \frac{\prod_{i=0}^{n-1}\left(1+\frac{s}{z_{i}}\right)}{\prod_{i=0}^{n}\left(1+\frac{s}{p_{i}}\right)}$

being $p_{T}$ a gain factor. The coefficients are chosen to set a maximum deviation in magnitude of $y$ decibels with respect to the ideal response. The intermediate required values are

$a=10^{\frac{y}{10(1-\alpha)}}$

$b=10 \frac{y}{10 \alpha}$

$c=10 \frac{y}{10 \alpha(1-\alpha)}$

being the poles and the zeros of the approximation

$p_{0}=p_{T} \sqrt{b}$

$p_{i}=p_{0}(a b)^{i}$

$z_{i}=a p_{0}(a b)^{i}$

The number of zeros and poles is related to the bandwidth

$N=\frac{\log \left(\frac{\omega_{\max }}{p_{0}}\right)}{\log (a b)}+1$

All the mentioned continuous time approximations have been compared in Fig. 9 in order to select those more suitable for control purposes. The comparison is based on the CL transfer function from (7), with the parameters detailed in Table 1 , setting $\alpha$ to 1.5 . Table 2 shows the parameters used for each approximation.

It can be seen that in terms of overshoot, the best ones are CFE and Chareff's approximations. In terms of gain bandwidth, the best ones are CFE, Chareff's and Matsuda's approximations. Finally, in terms of phase all options offer good response up to $1000 \pi \mathrm{rad} / \mathrm{s}(500 \mathrm{~Hz}$ ), but it could be assumed that Carlson's approach is the less convenient option. Then, hereinafter, the Chareff's approximation is considered as the best alternative.

\section{Performance of the fractional proportional-resonant controller}

This section analyses the steady-state frequency response differences between the conventional PRHC and the proposed FPR controller. The tuning procedure proposed in Section 3.4 is applied and the results obtained are discussed. Also, a general comparison between the most common controllers is addressed at the end of this section.

\subsection{Steady-state frequency comparison}

Fig. 10 shows a frequency comparison (steady-state analysis by means of their Bode plot) between an FPR and a PRHC controller compensating for the odd harmonic components up to the 7th. The different PRHC $k_{\text {Ih }}$ gains can be obtained from Table 1 considering that 


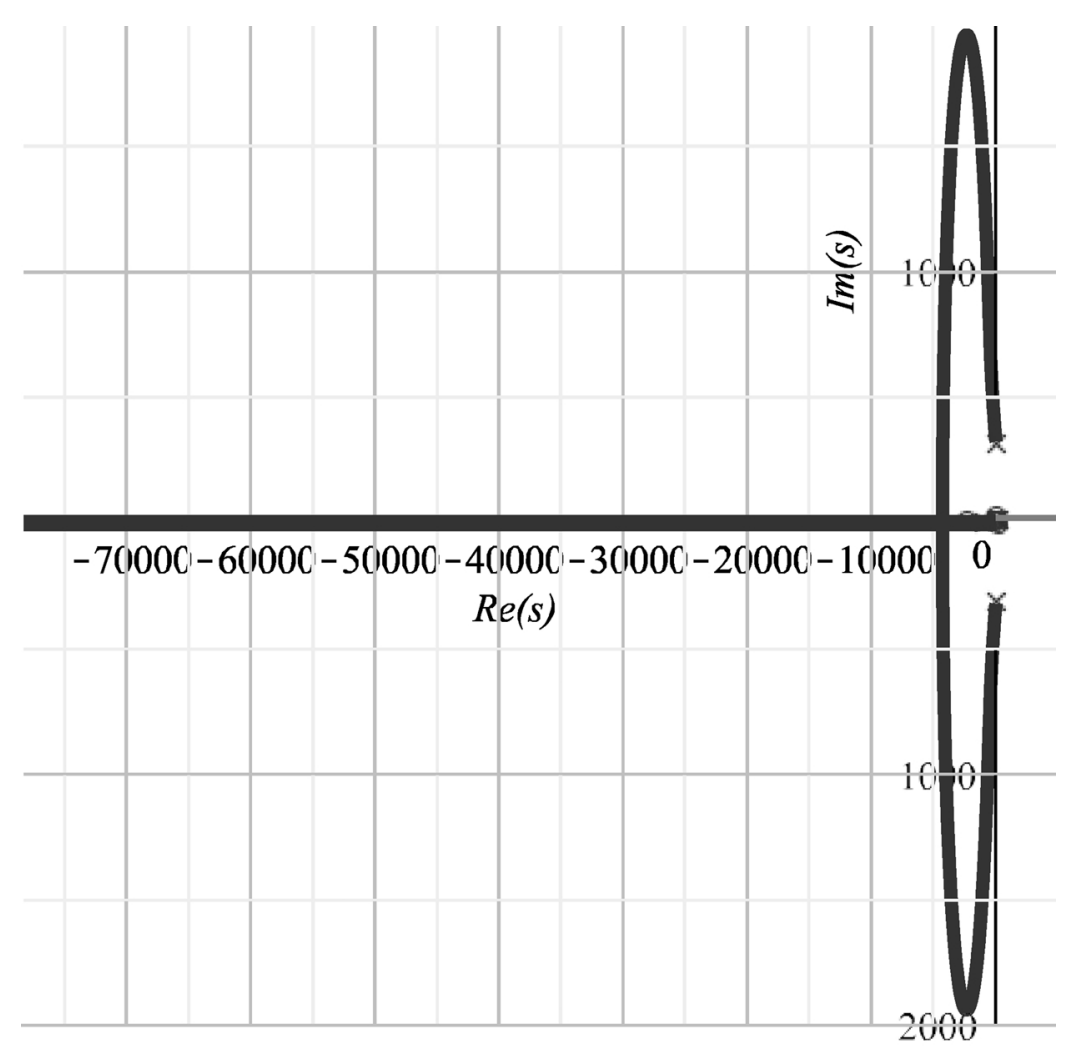

(a) Distant view

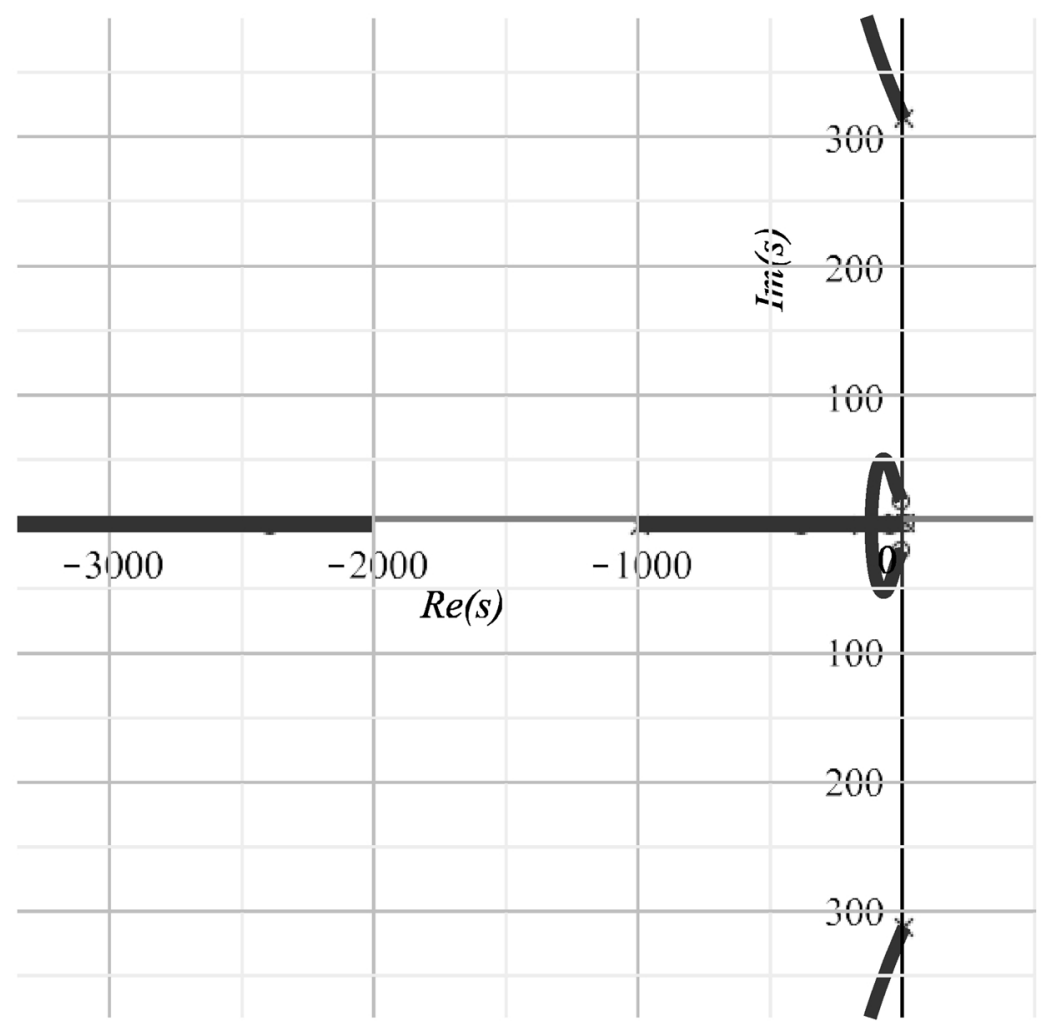

(b) Zoom

Fig. 14. Root locus. The $K$ factor that multiplies the open loop transfer function is swept from 0 to 5 . 


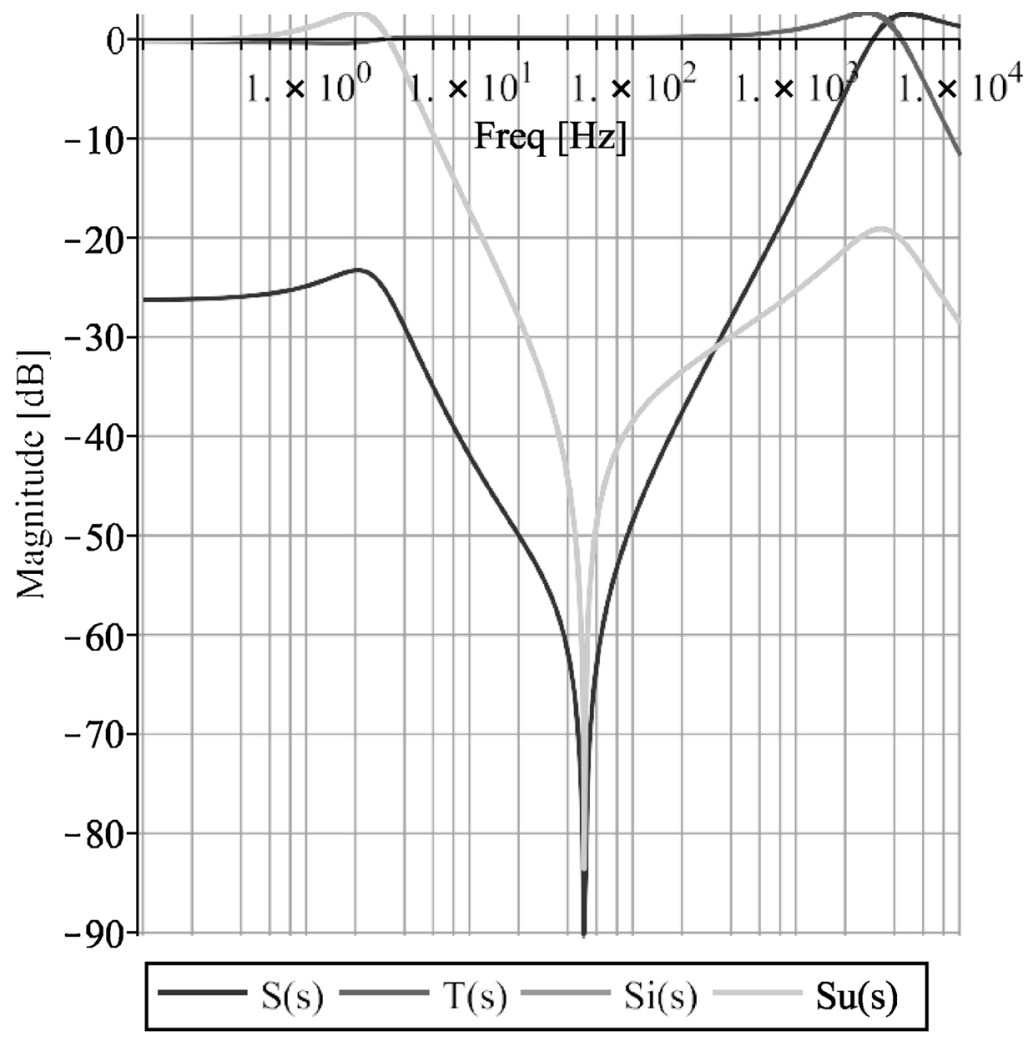

(a) Gain versus frequency

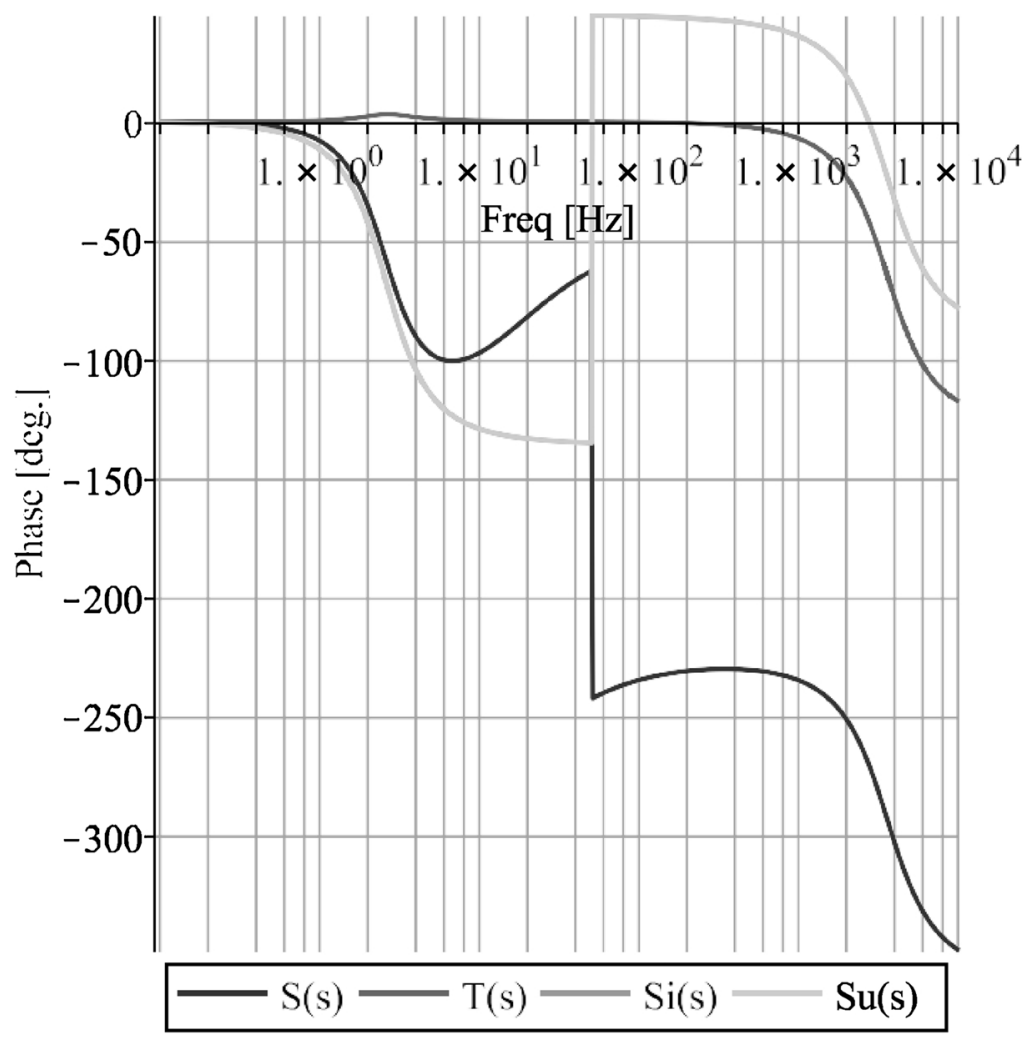

(b) Phase versus frequency

Fig. 15. Sensitivity analysis. 


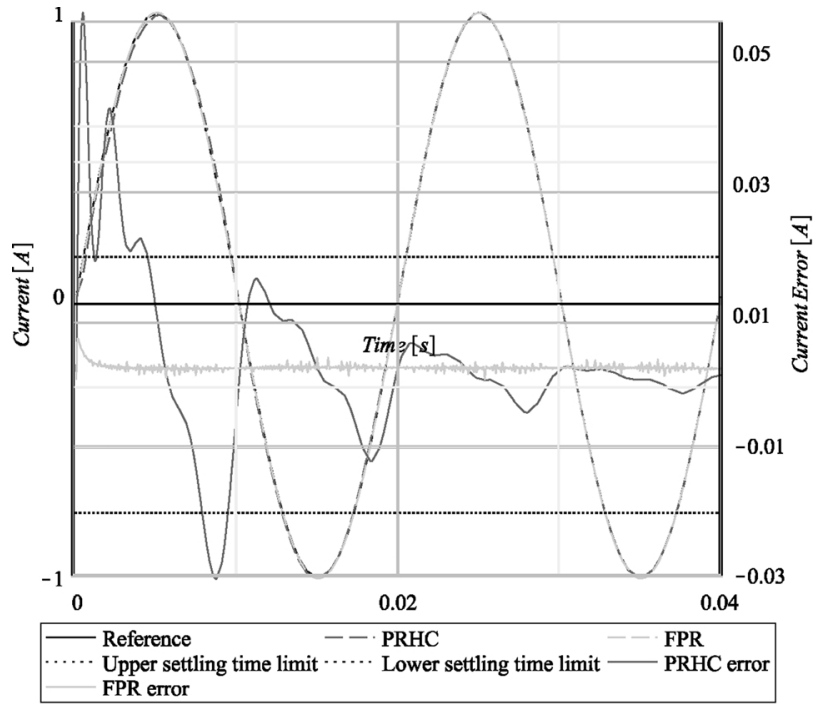

(a) $i_{L}^{*}=\sin \left(\omega_{0} \mathrm{t}\right)$

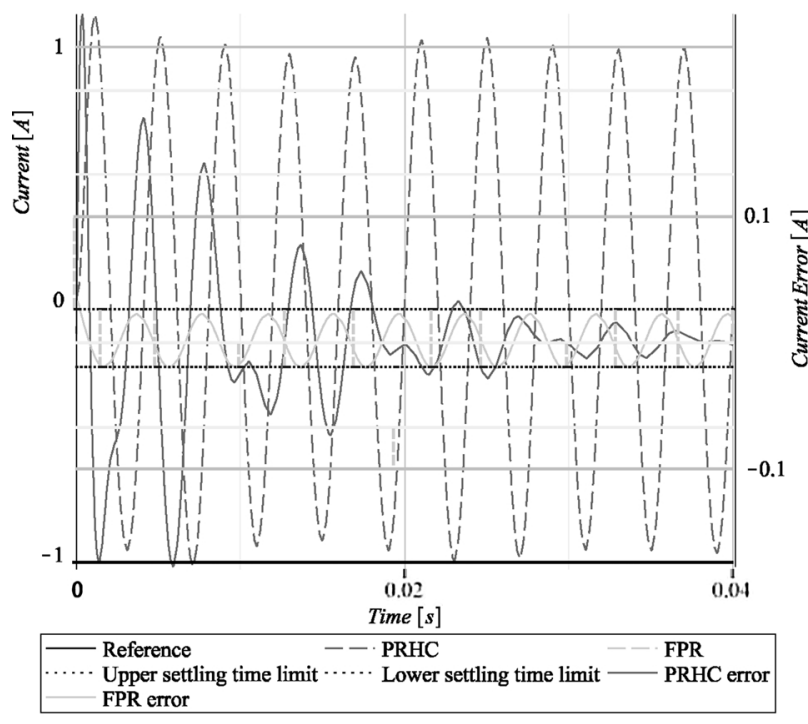

(c) $i_{L}^{*}=\sin \left(5 \omega_{0} \mathrm{t}\right)$

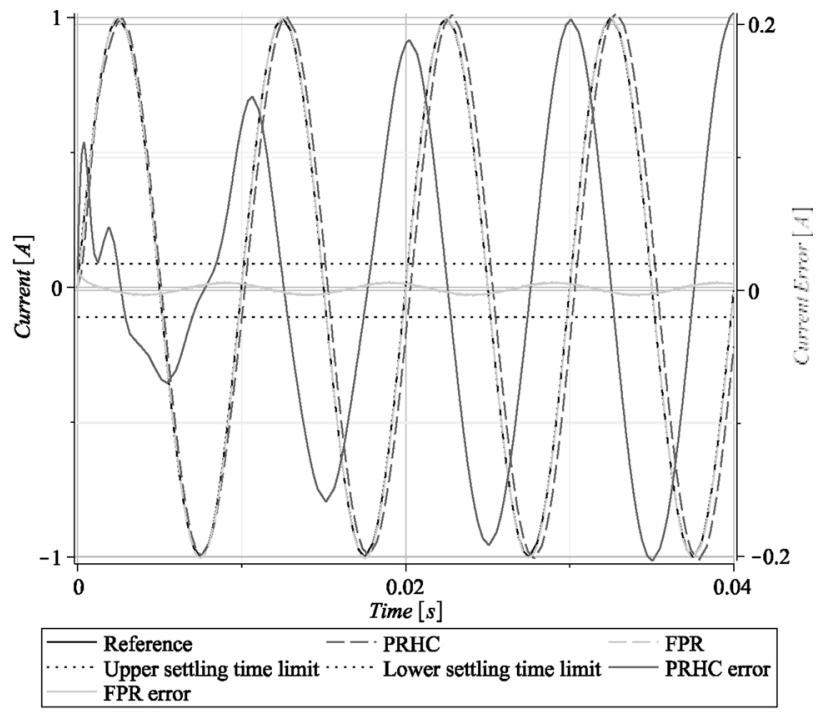

(b) $i_{L}^{*}=\sin \left(2 \omega_{0} \mathrm{t}\right)$

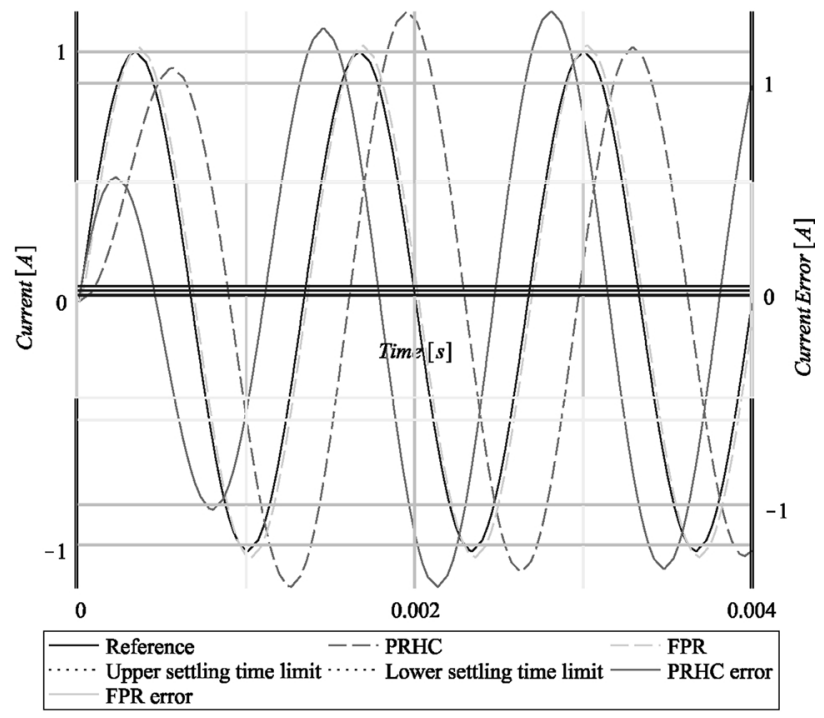

(d) $i_{L}^{*}=\sin \left(15 \omega_{0} t\right)$

Fig. 16. Time-response comparison when different harmonic components are regulated, being $\omega_{0}=100 \pi \mathrm{rad} / \mathrm{s}$.

$k_{I h}=k_{I} / h$. As can be seen, the use of an standard PRHC controller results in inter-harmonic excitation and in a high order transfer function. It should be highlighted that the inter-harmonic frequency excitation issue is reduced with the FPR controller. This is a direct consequence of the tuning procedure detailed in Section 3. As there is only one infinite gain from the inherent PR behaviour, the inter-excitation phenomena is suppressed. On the other hand, frequencies different than $\omega_{0}$ do not present pure zero steady-state error. However, depending on the $\alpha$ value, the error is low.

\subsection{Applying the tuning procedure}

This section aims to illustrate an example according to the tuning procedure exposed in Section 3.4, assuming $\omega_{0}=100 \pi \mathrm{rad} / \mathrm{s}(50 \mathrm{~Hz})$ :
1. Tune PR. Following [36] and considering the base values presented in Section 3 (see also Table 1), the obtained PR gains are $k_{p}=1.44$ and $k_{i}=4.28$.

2. Plot CL transfer function with FPR. Fig. 6(a) and (b) have illustrated how the gain and gain shift changes according to the angular frequency. However, only the frequency bandwidth up to $1000 \mathrm{~Hz}$ will be considered in the example. For the sake of clarity, Fig. 11 shows a zoom on this frequency range.

3. Select $\alpha$. A maximum theoretical delay of $6^{\circ}$ for the 15 th harmonic is desired. Thus, in Fig. 11 it is possible to see a marker (black circle) that indicates that $\alpha=1.5$ is a proper candidate.

4. Find an implementation for the FPR transfer function. Several references propose different approximations of fractional order operators [40-42]. Fig. 12 shows a Bode diagram comparison of (7) 


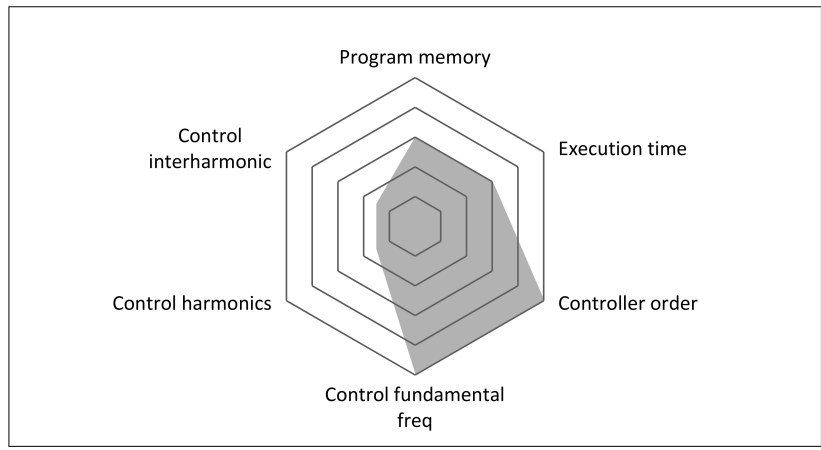

(a) PI controller

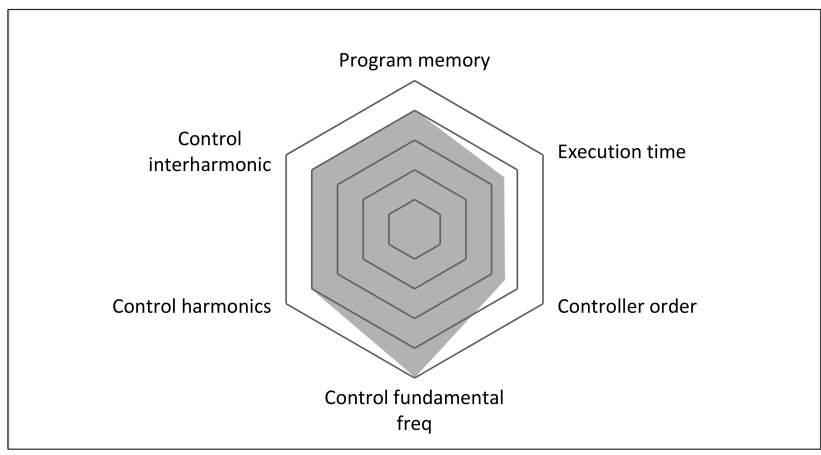

(c) FPR controller

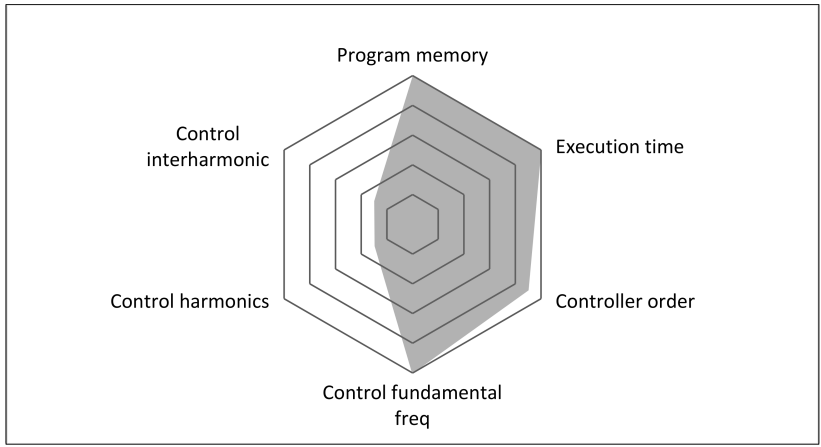

(b) PR controller

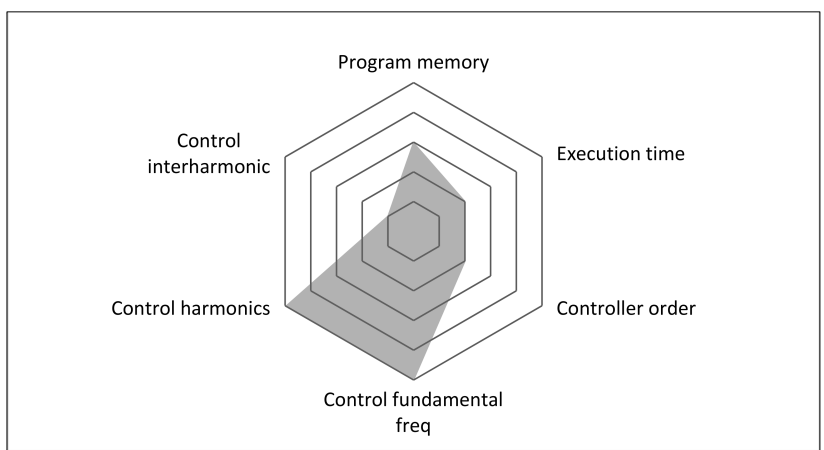

(d) PRHC controller

Fig. 17. Controller type comparison. Implementation and control criteria.

between the theoretical and the Chareff's approximation, being $p_{T}=1, y=2 \mathrm{~dB}$ and $n=4$, yielding the $s^{-0.5}$ approximation

$$
\begin{aligned}
s^{-0.5} \approx \frac{6.3 \times 10^{-8} s^{4}+0.74 \times 10^{-4} s^{3}+0.12 \times 10^{-1} s^{2}+0.29 s+1}{1.0 \times 10^{-9} s^{5}+0.30 \times 10^{-5} s^{4}+0.12 \times 10^{-2} s^{3}} & \\
& +0.77 \times 10^{-1} s^{2}+0.75 s+1
\end{aligned}
$$

Consequently, the FPR(s) is approximated as

$F P R(s) \approx \frac{0.14 \times 10^{-5} s^{6}+0.40 \times 10^{-2} s^{5}+1.6 s^{4}+110 s^{3}+2700 s^{2}+42000 s+1.3 \times 10^{5}}{\left(s^{2}+96000\right)\left(6.3 \times 10^{-8} s^{4}+0.75 \times 10^{-4} s^{3}+0.12 \times 10^{-1} s^{2}+0.30 s+1\right)}$

5. Robustness analysis. Fig. 13 depicts a zero-pole map when the system parameters base values are multiplied by a scale $C$ factor from 0.2 to 2 in steps of 0.2 . The bigger black cross/circle indicates $C=1$, dark grey $C<1$, and light grey $C>1$. It is observable that for sensible tolerance errors, although the poles are displaced, no unstable situations are achieved. $C<1$ values displacement reduce the imaginary and the real parts. It can be also seen that the variation of $R$ practically does not affect the zero-pole map.

The root locus graph is shown in Fig. 14. It can be seen that the direct control chain gain can be increased or decreased without compromising the stability of the case study.

Finally, Fig. 15 shows a complete sensitivity analysis. For the angular frequencies up to $1000 \pi \mathrm{rad} / \mathrm{s}(500 \mathrm{~Hz})$, it can be seen that only $S(s)$ presents an excitation region corresponding to the sensitivity to disturbances in the $i_{L}$ current. This kind of uncertainty is unlikely to be high unless coupled inductances or other electromagnetic emitter generators are close to the inverter.
According to the analysis developed, the FPR is considered robust enough.

6. Simulations. When a PRHC is used, the controllable harmonic components depend on the existence of the corresponding HC controller in parallel with the fundamental one (see (3)), considering the 3rd, 5th and 7th harmonics, the following FPR controller is obtained:

$$
\begin{aligned}
G_{P R H C}(s)= & 1.44+4.28 \omega_{0} s\left(\frac{1}{s^{2}+\omega_{0}^{2}}+\frac{1 / 3}{s^{2}+9 \omega_{0}^{2}}+\frac{1 / 5}{s^{2}+25 \omega_{0}^{2}}\right. \\
& \left.+\frac{1 / 7}{s^{2}+49 \omega_{0}^{2}}\right)
\end{aligned}
$$

This means that if a different harmonic component appears, the PRHC is not able to track it with zero steady-state error, especially in terms of phase lag. In Fig. 16, the CL time response of a PRHC up to the 7th harmonic and for a FPR using Chareff's approximation is compared. Moreover, the current error is also shown in the timedomain plots for different harmonic references. Note that the reference current is $1 \mathrm{~A}$ in all cases. On one hand, even though the PRHC response presents some tracking capability, refer to Fig. 16(a) and (c), the FPR offers a more accurate tracking behaviour, not only remarkable for harmonic components over the 7th, as can be observed in Fig. 16(d) but also for inter-harmonics, as depicted in Fig. 16(b).On the other hand, in Fig. 16(a) it can be seen that for the conventional PR formulation, the error is within a threshold of $\pm 2 \%$ in the settling time proposed in Table 1 , that corresponds to $\mathrm{ms}$. In the case of the FPR the settling time has been reduced, mainly due to a good tracking capability for more frequencies. Note that under sinusoidal references, the error is changing from positive to 


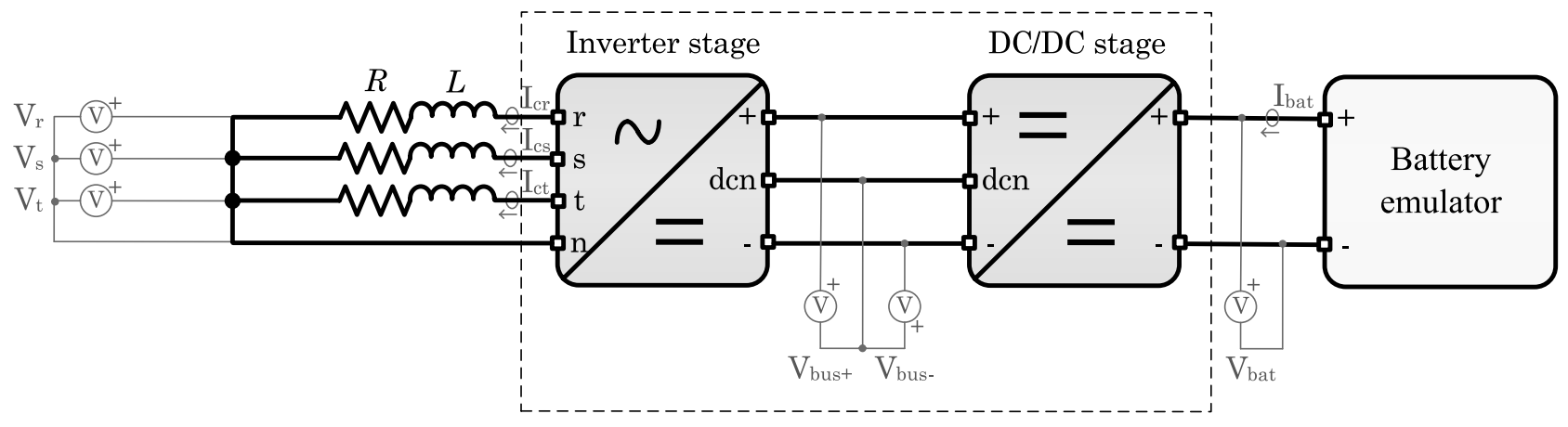

(a) Short-circuited configuration

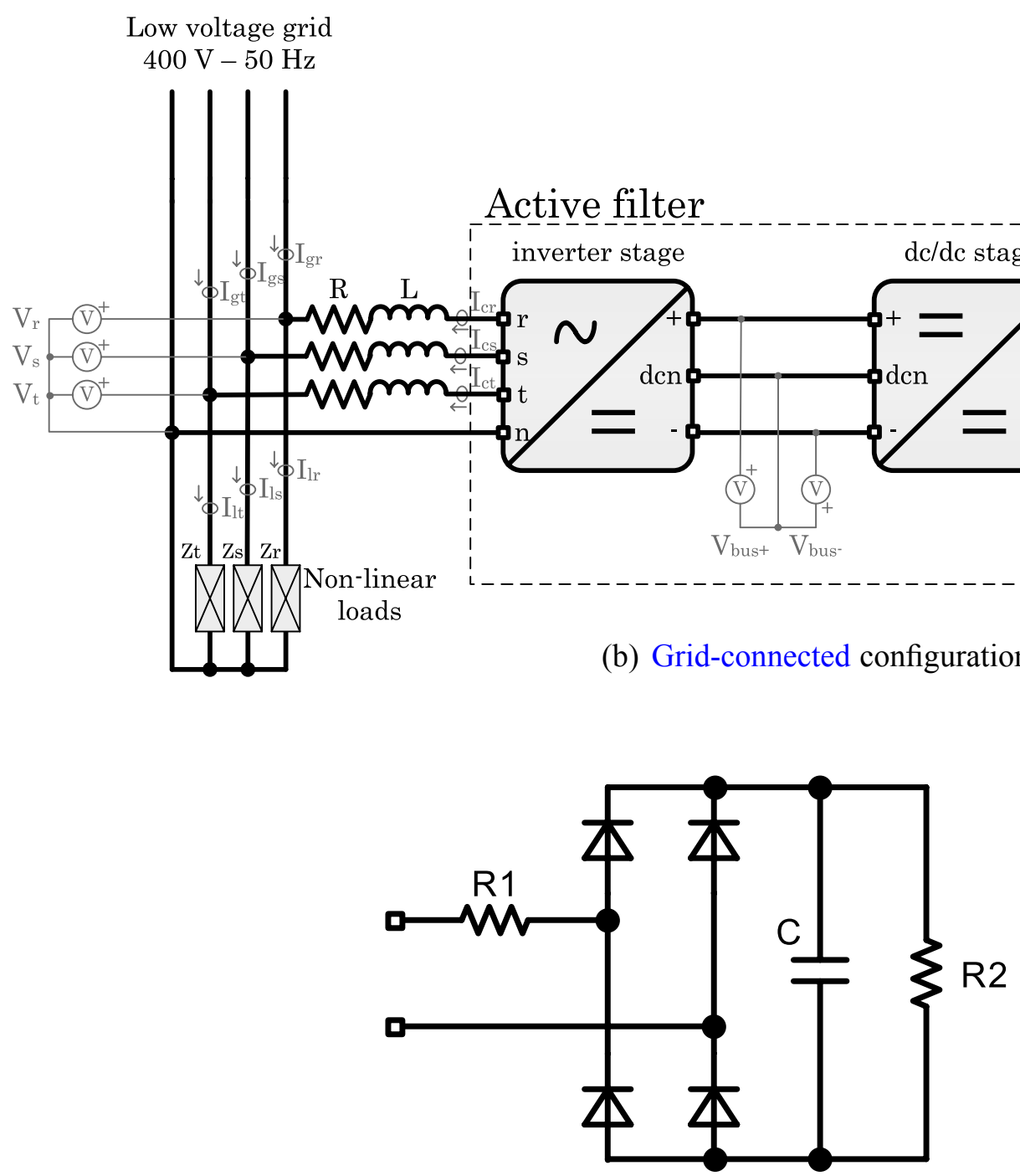

(c) Proposed non-linear load for active filtering operation

Fig. 18. Set-up scheme

negative due to the reference waveform itself. This changing behaviour propitiates that in practice, more than just the desired component have to be considered.It should be remarked that (24) has a characteristic equation of 8th order, whereas (6) is a 6th order equation. Then, it can be stated that FPR offers extended tracking capabilities even with a lower order transfer function compared to PRHC. This fact implies a lower computational burden, as will be shown in Section 4.3 - Fig. 17. 


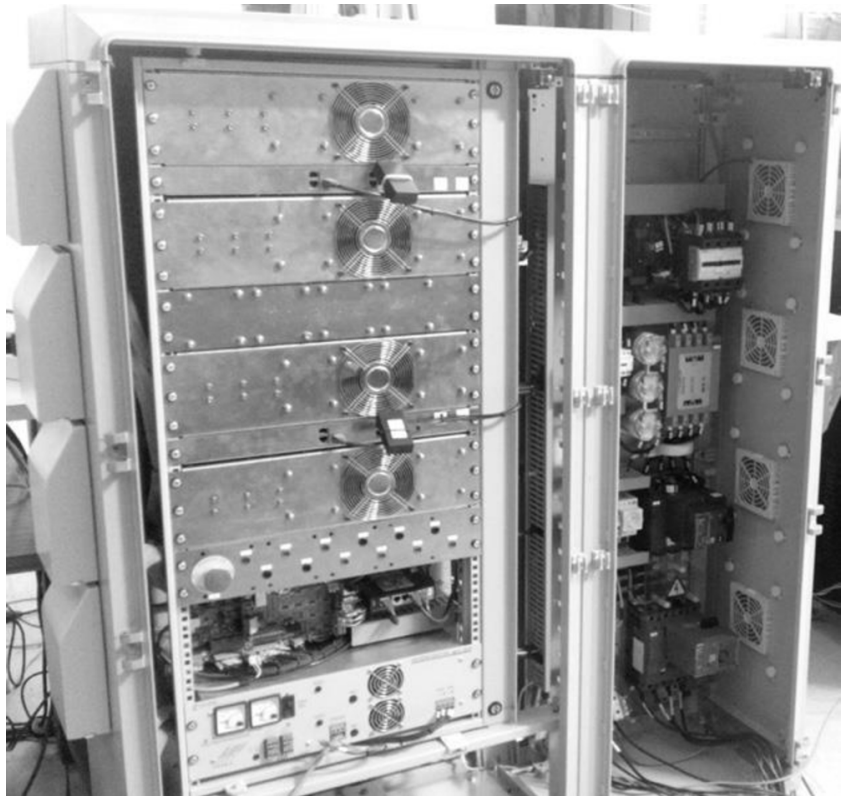

Fig. 19. Experimental power converter. Two three-phase full-bridge CCS050M12CM2 converters are in parallel for the DC/DC stage and two more for the four-wire inverter.

\subsection{General comparison}

After the different steps followed in previous sections and the obtained results, this section aims to present a summary comparison contrasting the advantages and drawbacks of different controller type options; PI, PR, PRHC and FPR. Thus, Fig. 17 accounts for a the comparison based on control and implementation criteria. For more details refer to Table 3 in Appendix.

From Fig. 17 it can be observed that each one of the four compared controllers has a different distribution area that explains its different properties, and its strong and weak points. While the PI controller has the lowest order of all them, being the easiest to implement and the most robust the necessity to apply mathematical transformations to operate in a synchronous reference frame, penalizes the memory required and the execution time. Furthermore, apart from the fundamental frequency, its behaviour compensating harmonics is deficient. In contrast, the PR controller with a similar control behaviour is suitable for low computational burden system applications, being the controller with the lowest execution time and program memory usage. However, adding harmonic controllers, the resulting PRHC controller improves greatly the control of the harmonics but losses its main advantage in the implementation field.

On the contrary, the new FPR formulation is a good trade-off for such applications that requires to manages to control several harmonic components without employing high order controllers, as could be the case of active filters or high accuracy AC current feeders. Usually, in this kind of applications it is necessary to adapt the code in order to obtain the desired multi-harmonic tracking control. This implies that memory and time execution can be crucial depending on the type of controller used, mainly for the case of low cost processors.

\section{Experimental results}

This section presents the implementation of the FPR controller developed in Section 4.2. The FPR controller has been implemented in a
Texas Instruments DSP, specifically Concerto F28M35, and it has been tested with a $20 \mathrm{kVA}$ full Silicon-Carbide MOSFET (CCS050M12CM2) based power converter. Such a test platform consists of a three-phase four-wire inverter with split DC-link and a three-leg DC/DC stage, as shown in Fig. 18. The DC/DC converter steps up the battery emulator voltage from 400 VDC to 800 VDC and balances the two semi-buses of the DC-link. A Sinusoidal Pulse Width Modulation (SPWM) strategy for the four-wire three-leg inverter is applied. The sampling and switching frequency are set to $30 \mathrm{kHz}$. All experimental plots (Figs. 20-26) have been obtained acquiring real data at $1.25 \mathrm{MHz}$ from a DL9040 Yokogawa oscilloscope. All plots have been depicted using Matlab.

A first short-circuited inverter-based configuration (Fig. 18(a)) is considered in order to show the multi-harmonic tracking capability of the FPR controller. In this sense, the inverter coupling filter (L-type) is short-circuited so the plant is not altered by a $v_{c}$ voltage disturbance (Fig. 5). As this simple configuration is only intended to verify the theoretical tracking capability response, a second case with the converter operating as an active filter is presented afterwards. In such a case (Fig. 18(b)), the AC coupling filter is connected to a low-voltage $400 \mathrm{~V}-50 \mathrm{~Hz}$ grid in which a strong non-linear load, as the one shown in Fig. 18(c) is used.

A picture of the converter set-up can be seen in Fig. 19. The experimental parameters of the plant are $L=490 \mu \mathrm{H}$ and $R=45 \mathrm{~m} \Omega$. The controller presented in (23) has been implemented, per phase, in the discrete time domain applying Tustin discretization.

For the short-circuited inverter-based configuration case (Fig. 18(a)), a complex multi-harmonic reference is generated. Phase $r$ is used to generate the positive part of about $50 \mathrm{~ms}$ period "Batman" signal composed by inter-harmonics. In addition, the experiment is repeated using the PRHC presented in (24) in order to compare the behaviour of the two controllers as in the Section 4.2.

In Fig. 20(a), the Fast Fourier Transforms (FFT) of the reference A(t) and both controllers are represented, thus detailing the multi-harmonic content. It can be seen that the harmonic content in the region between 0 and $800 \mathrm{~Hz}$ is tracked almost perfectly by the FPR controller. In contrast, the FPR has a relative tracking capability but presents error in all the frequencies, due to the fact that it has an acceptable tracking capability for this range of frequencies. However, it presents a small error in all of them. In the region between 1200 and $2800 \mathrm{~Hz}$, the FPR shows a small excitation. Fig. 20(b) shows a capture in which it is demonstrated that the FPR controller is able to track complex inter-harmonics set-points with better results than the PRHC controller.

To demonstrate the FPR tracking capability with another example, a multi-harmonic reference with odd fundamental harmonics up to the 11 th is generated. The set-up used has a rated current of $20 \mathrm{~A}$. The harmonic content of the reference and the controllers response are shown (in per unit) in Fig. 21(a). It can be seen that the tracking capability of both controllers is similar up to the 7th harmonic. For higher harmonics, the PRCH controller is not able any more to follow properly the reference, making necessary the addition of more compensators. In contrast, the FPR shows a certain capability to follow partially these frequencies. In Fig. 21(b), it its shown that the FPR controller tracks properly the mentioned reference with a significantly smaller error than the PRHC compensator.

Finally, using the short-circuit set-up, a last test is done to compare the transient behaviour between the two controllers. This test aims to validate the simulations results obtained in Fig. 16. For this purpose, different harmonics are considered in the reference signal in order to check both the transient and the steady state response. Figs. 22 and 23 show, respectively, the different references and the real currents in pu (base current $40 \mathrm{~A}$ ), and the resulting error. On one hand, it can be seen that the steady state error up to the 7 th harmonic is similar for both 

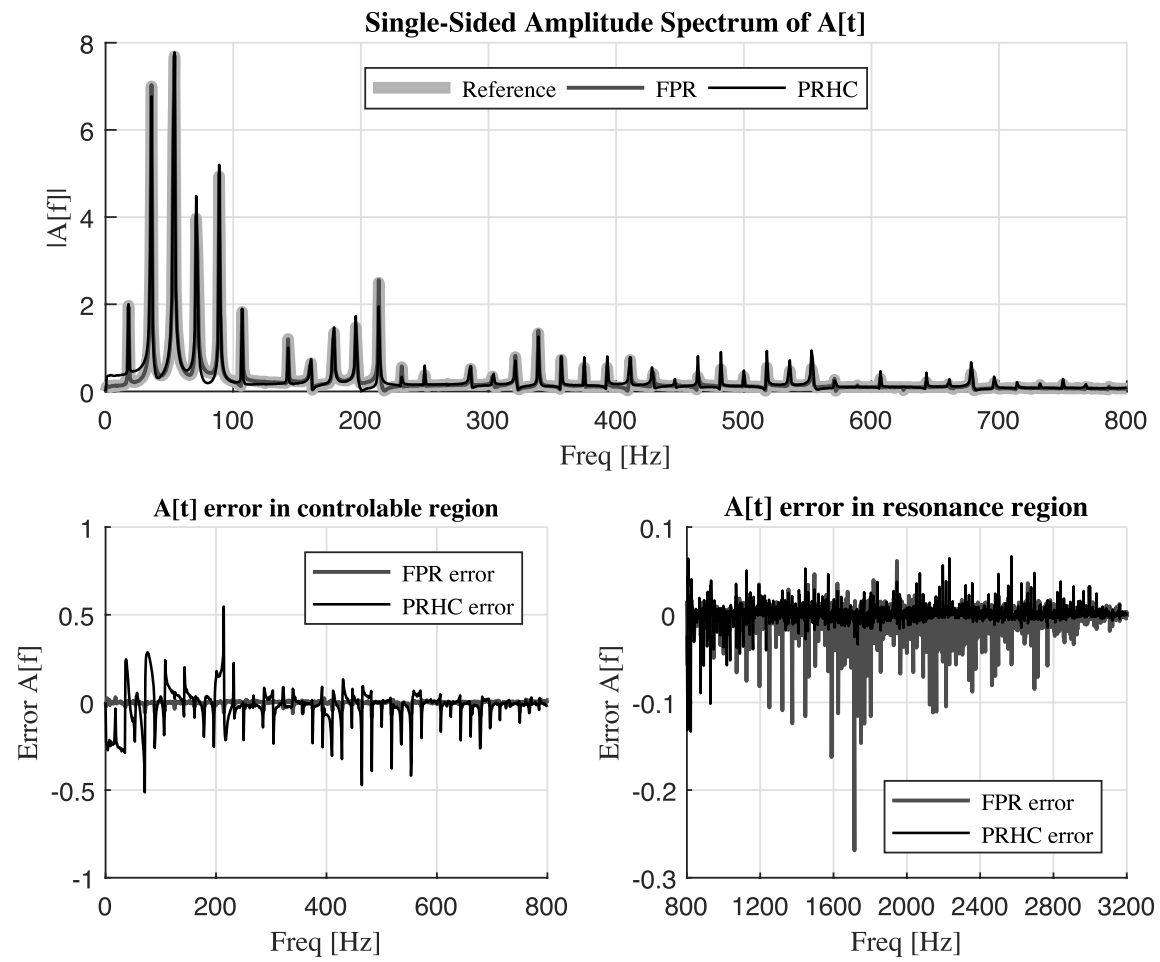

(a) FFT of the reference and the FPR and PRHC controller response
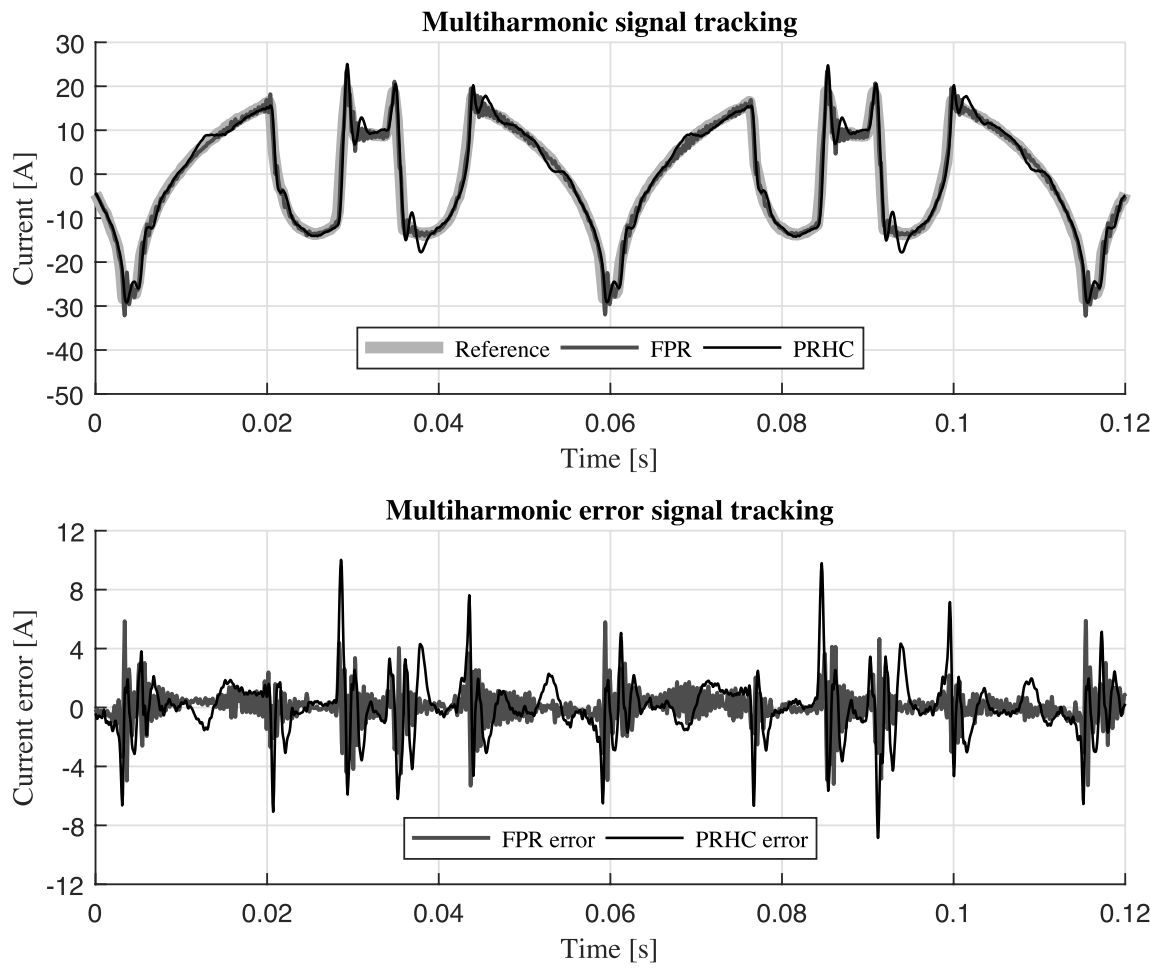

(b) Tracking response of the FPR and PRHC controller

Fig. 20. Multi-harmonic ("Batman") signal. 

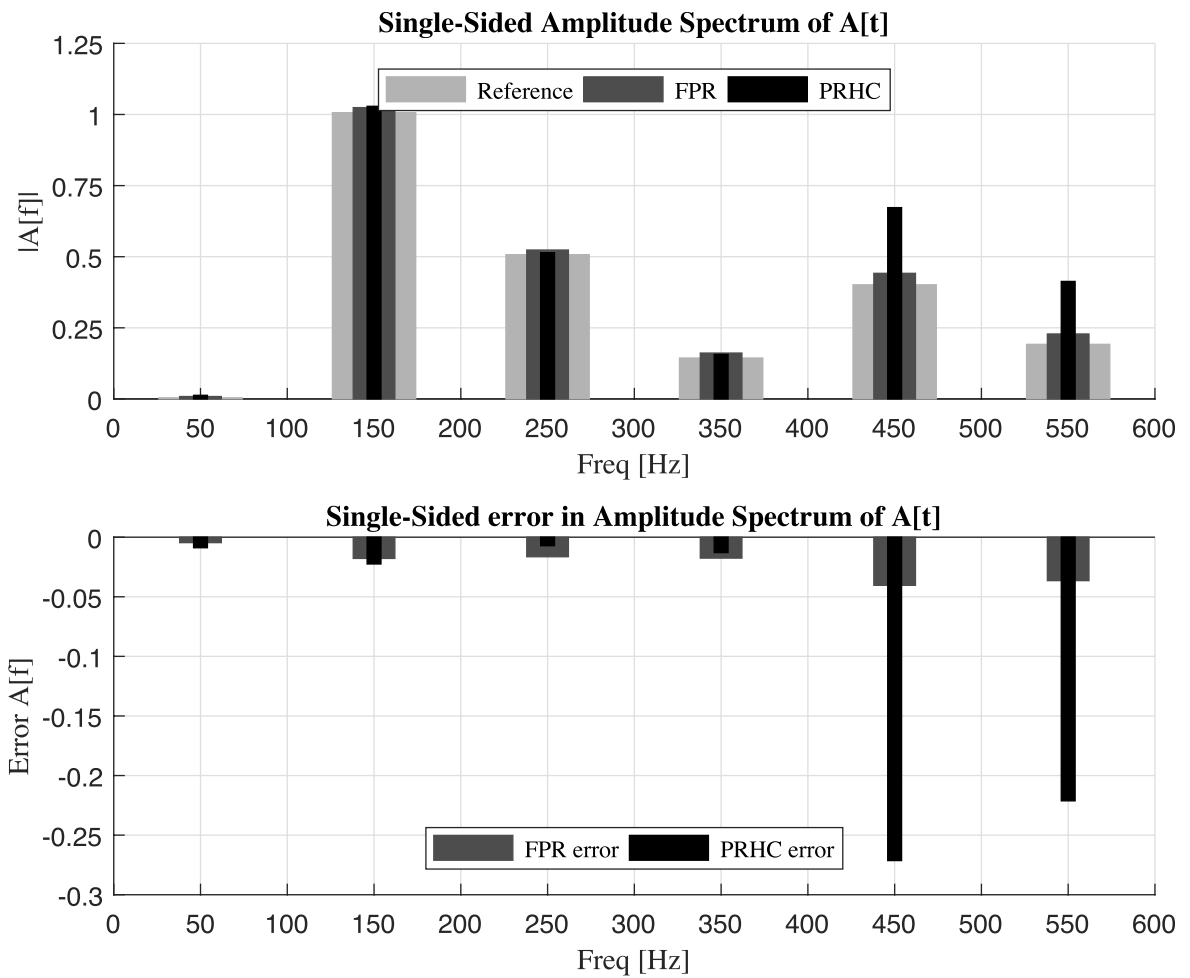

(a) FFT of the reference and the FPR and PRHC controller response
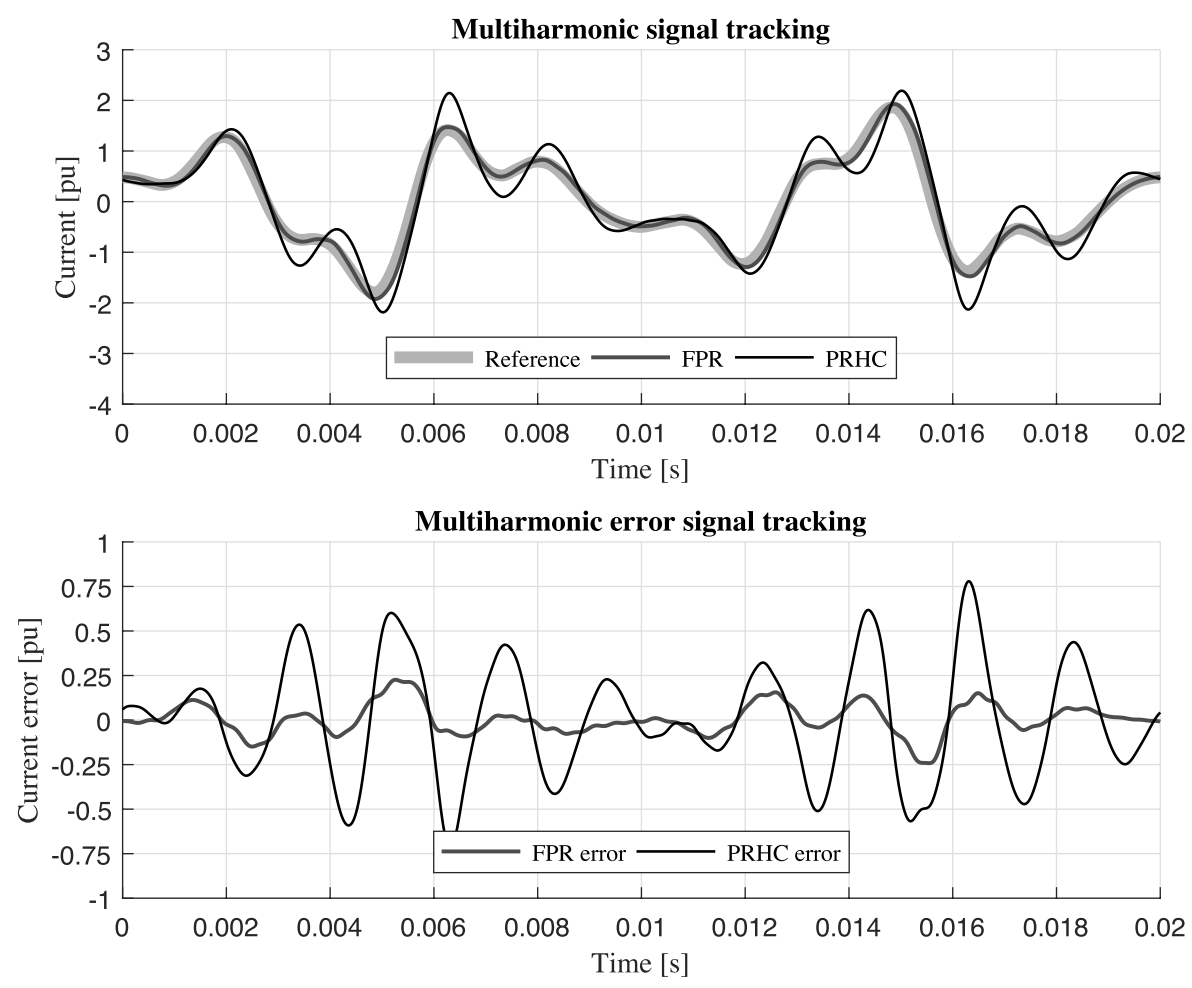

(b) Tracking response of the FPR and PRHC controller

Fig. 21. Multi-harmonic signal. 


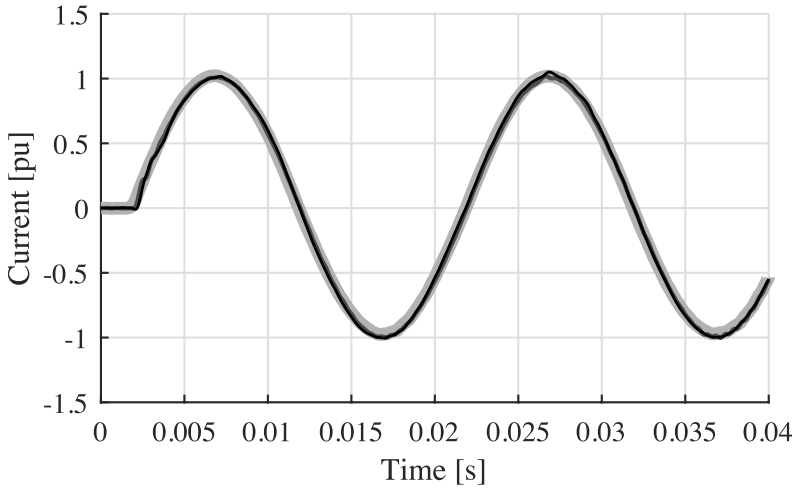

Reference $\longrightarrow$ FPR $\longrightarrow$ PRHC

(a) $i_{L}^{*}=\sin \left(\omega_{0} \mathrm{t}\right)$

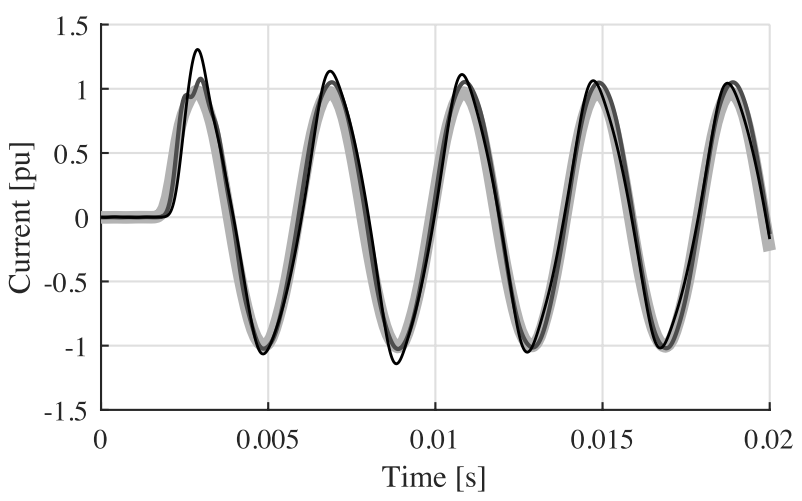

Reference $\longrightarrow$ FPR $\longrightarrow$ PRHC

(c) $i_{L}^{*}=\sin \left(5 \omega_{0} \mathrm{t}\right)$

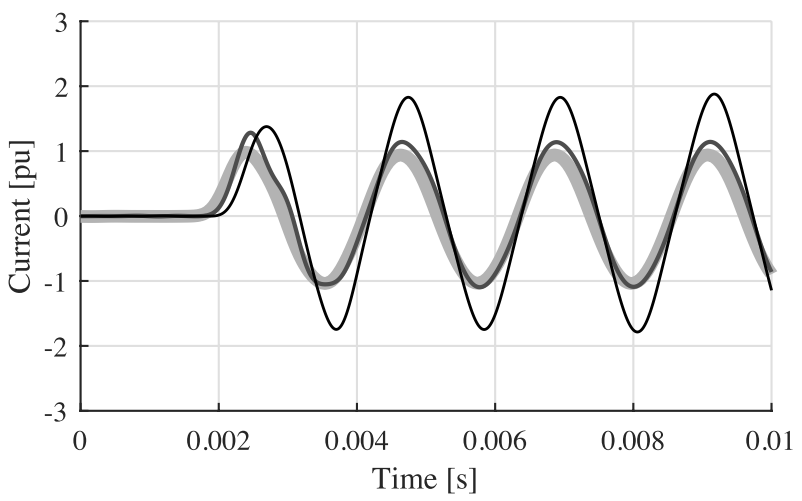

Reference $\longrightarrow$ FPR PRHC

(e) $i_{L}^{*}=\sin \left(9 \omega_{0} \mathrm{t}\right)$

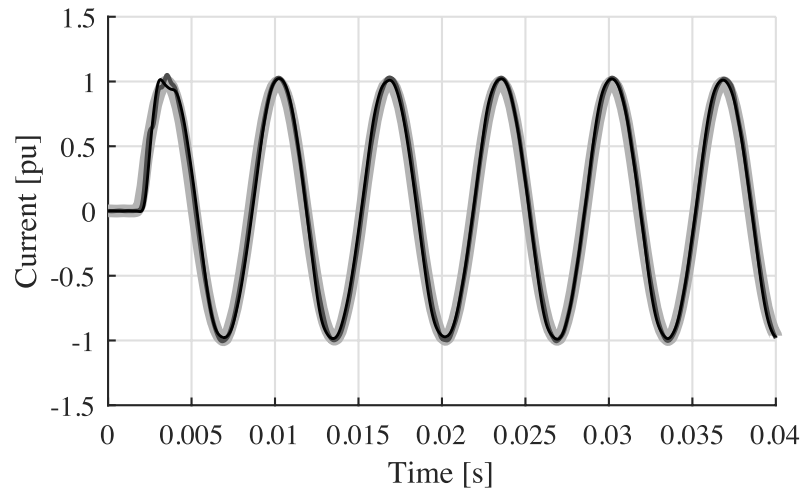

Reference $\longrightarrow$ FPR $\longrightarrow$ PRHC

(b) $i_{L}^{*}=\sin \left(3 \omega_{0} \mathrm{t}\right)$

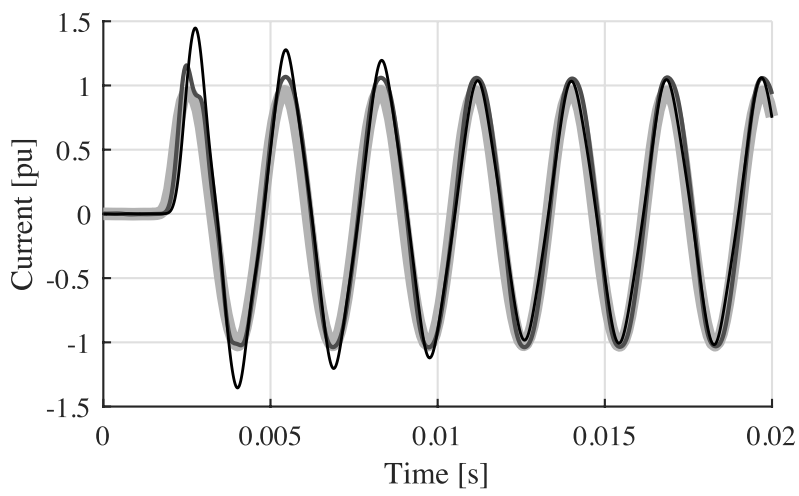

Reference $\longrightarrow$ FPR $\longrightarrow$ PRHC

(d) $i_{L}^{*}=\sin \left(7 \omega_{0} \mathrm{t}\right)$

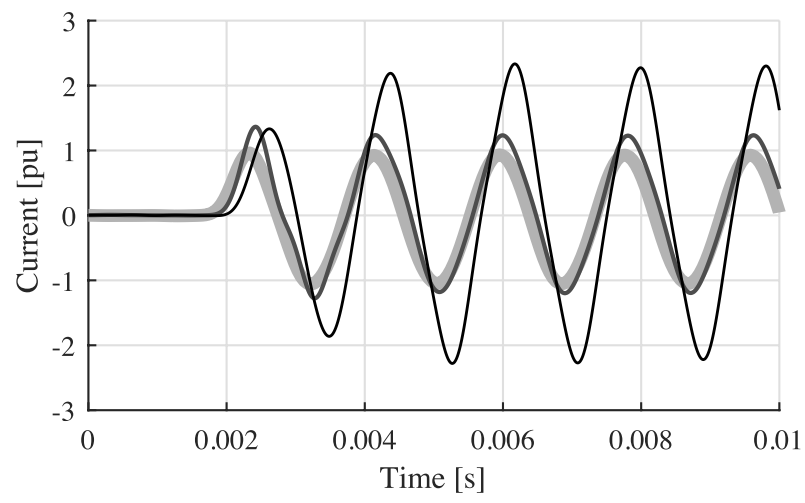

Reference

FPR $\longrightarrow$ PRHC

(f) $i_{L}^{*}=\sin \left(11 \omega_{0} \mathrm{t}\right)$

Fig. 22. Time-response tracking comparison when different harmonic components are regulated, being $\omega_{0}=100 \pi \mathrm{rad} / \mathrm{s}$. 


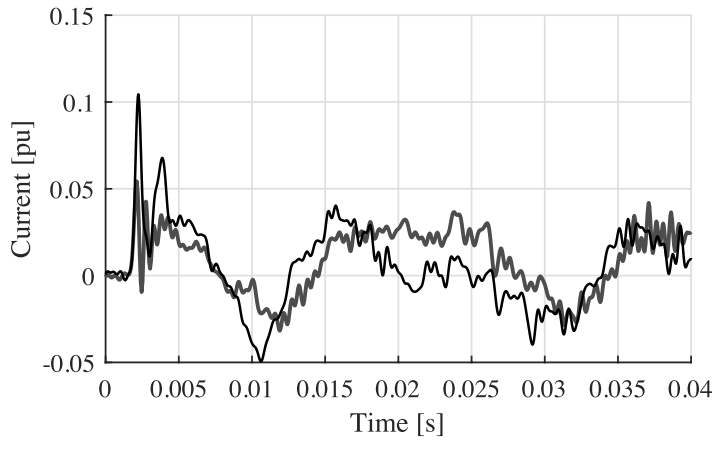

FPR error $\longrightarrow$ PRHC error

(a) $i_{L}^{*}=\sin \left(\omega_{0} t\right)$

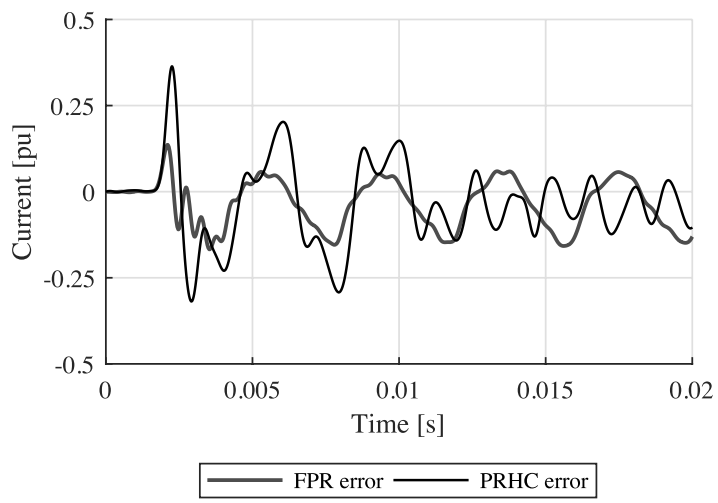

(c) $i_{L}^{*}=\sin \left(5 \omega_{0} \mathrm{t}\right)$

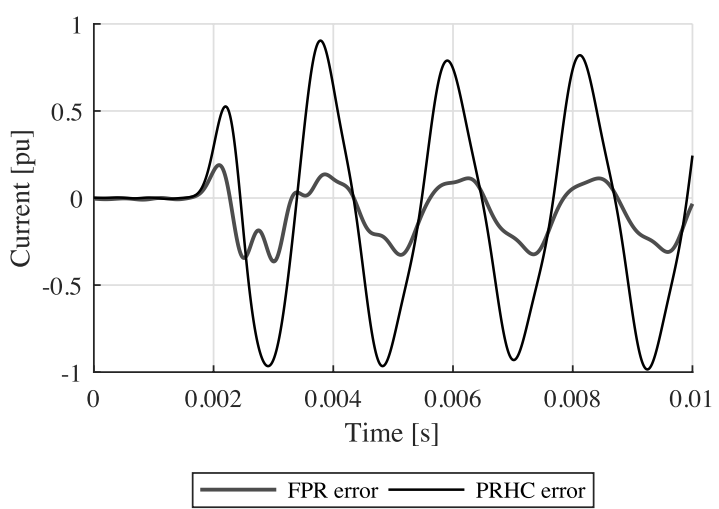

(e) $i_{L}^{*}=\sin \left(9 \omega_{0} \mathrm{t}\right)$

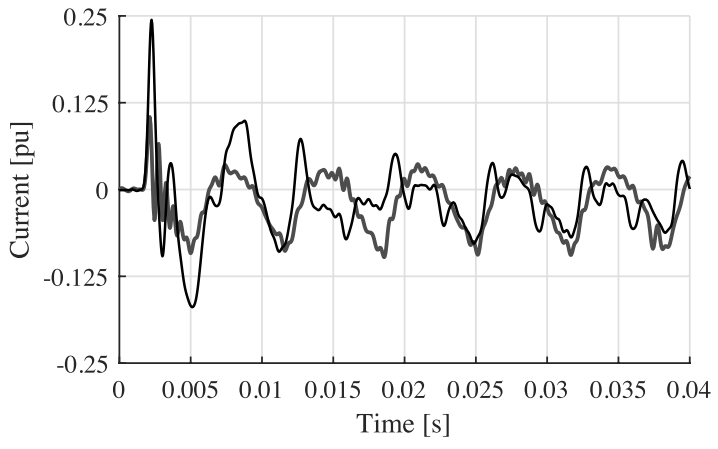

FPR error $\longrightarrow$ PRHC error

(b) $i_{L}^{*}=\sin \left(3 \omega_{0} \mathrm{t}\right)$

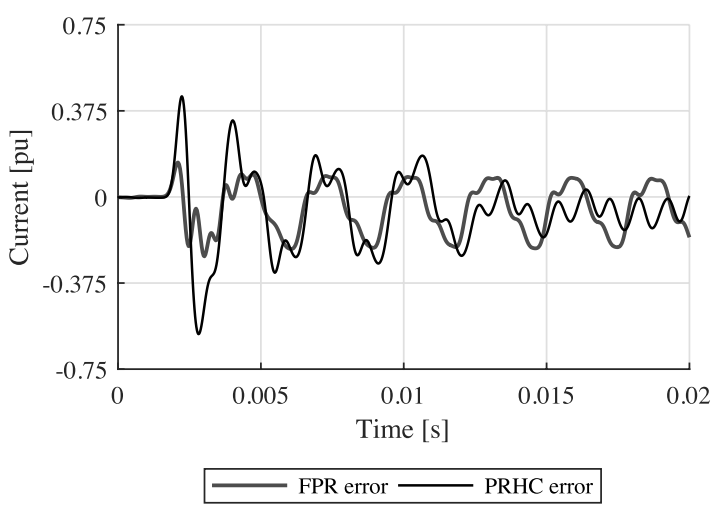

(d) $i_{L}^{*}=\sin \left(7 \omega_{0} \mathrm{t}\right)$

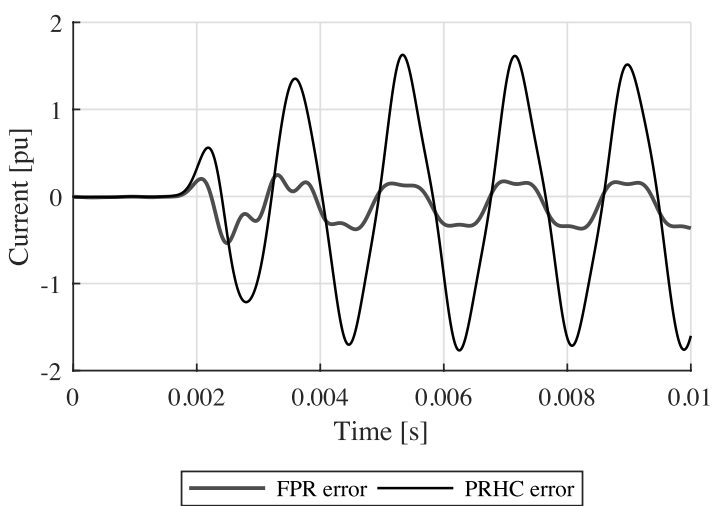

(f) $i_{L}^{*}=\sin \left(11 \omega_{0} \mathrm{t}\right)$

Fig. 23. Time-response error comparison when different harmonic components are regulated, being $\omega_{0}=100 \pi \mathrm{rad} / \mathrm{s}$.

controllers. For frequencies above the 7th harmonic, the PRCH is not able to properly follow the references, whereas the FPR presents a certain tracking capability. This is also represented in Fig. 24, where the amplitude spectrum of the reference and the two different responses are shown. On the other hand, the transient differences between both controllers are clearly noticeable in Fig. 23. In the case of the FPR controller, after one signal period the error is already in the steady state region. Alternatively, in the PRHC case, the transient lasts around three cycles.

On the other hand, the grid-connected case is sub-divided into two operations modes; one as an active filter and the other one delivering a $50 \mathrm{~Hz}$ current to a non-ideal grid. 

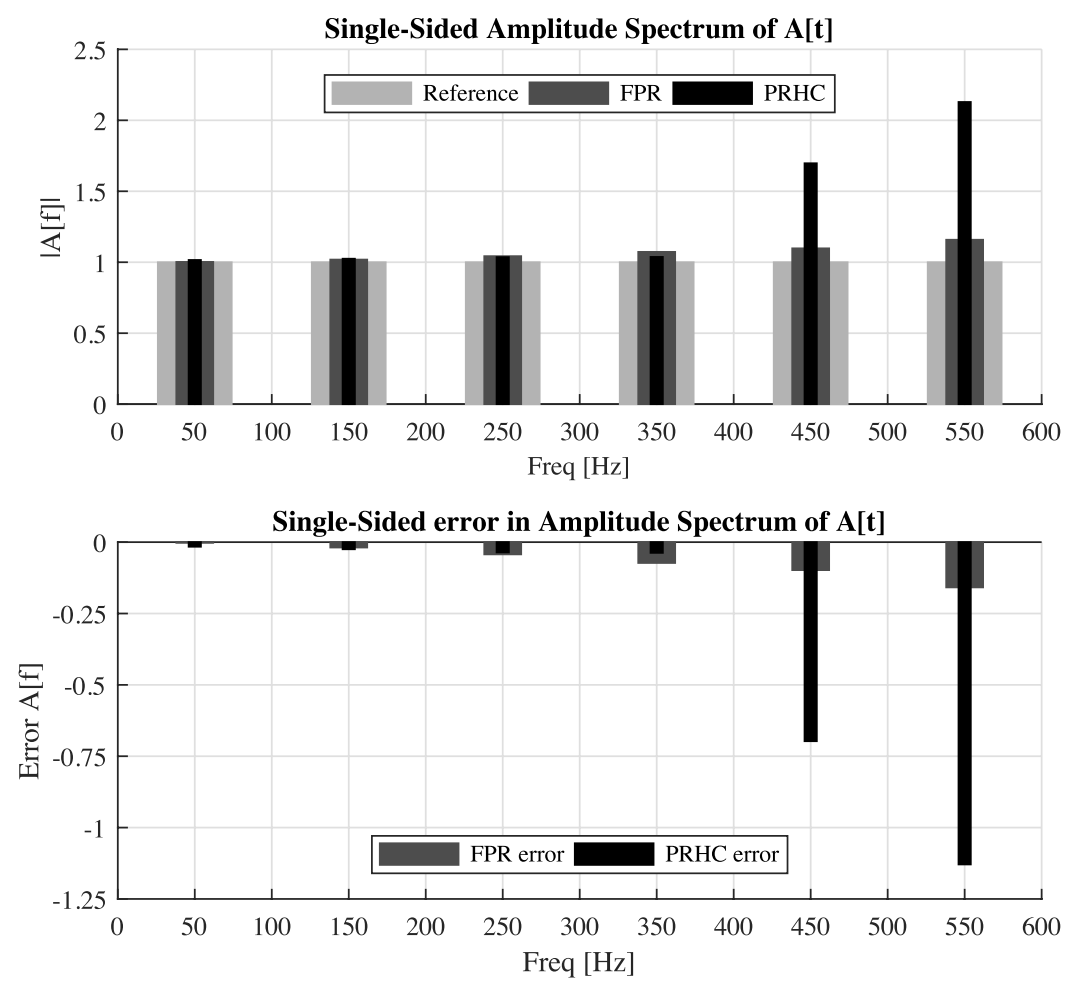

Fig. 24. Amplitude spectrum of the different references and the FPR and PRHC controller response.

For the active filtering operation case the results concerning the active phase $r$ can be seen in Fig. 25. The active filter role consists of delivering all the possible harmonic content (Note that the fundamental components will be delivered by the grid.). Thus, it can be observed that the controller is able to compensate for a high number of harmonic components. A strong single phase non-linear load, as the one depicted in Fig. 18(c), is connected between phase $r$ and the neutral wire, and it has the following values: $R_{1}=1 \Omega, R_{2}=22 \Omega$ and $C=2.2 \mathrm{mF}$. The total harmonic distortion (THD) of such a load $\left(\mathrm{I}_{\text {load }}\right)$ is $62.8 \%$. The controller is able to inject the major harmonic current components $\left(\mathrm{I}_{\text {conv }}\right)$, reducing the THD of the utility ( $\left.\mathrm{I}_{\text {grid }}\right)$ up to a $3.2 \%$. Note that the THD of $\mathrm{I}_{\text {conv }}$ (harmonic content delivered by the active filter) is approximately $7.2 \%$, which is significantly lower than $62.8 \%$. The reason for this to happen is that the fundamental component of this current is $150 \mathrm{~Hz}$ (it does not have a $50 \mathrm{~Hz}$ component because the first most significant harmonic component of the non linear load refers to the 3rd harmonic).

Lastly, to evaluate the high performance of the FPR controller under non-ideal grids, the FPR controller is compared with the PRHC controller presented in Eq. (24). This case study consists of delivering an ideal $50 \mathrm{~Hz}$ current to a non-ideal three-phase grid distorted by different harmonics components, as can be seen in upper sub-plots of Fig. 26. The performance of the current between both controllers is compared, delivering $5.75 \mathrm{~kW}$ per phase $(25 \mathrm{Arms}, 50 \mathrm{~Hz}$ current setpoint). In the bottom sub-plots it can be seen that the FPR offers similar performance at the fundamental component $(50 \mathrm{~Hz})$. As the PRHC has specific harmonic compensators at the 3rd, 5th, 7th harmonics presents lightly better results than the FPR. But, as the requirements at high frequency increases, the FPR contributes to enhance the current quality delivered to the grid. Note that the harmonics bars are multiplied by a factor of 50 for better visibility.

\section{Conclusion}

A novel controller based on the application of fractional order calculus into proportional-resonant controllers has been presented. Such a controller enhances the frequency tracking behaviour of AC references with a low order transfer function in comparison with the conventional multi-harmonic proportional-resonant controllers. In this sense, the fractional proportional-resonant (FPR) controller emerges as a good alternative to be implemented in systems in which computational time is critical and memory dependence is important. In this paper, the application analysed is the current control loop of a voltage source converter, and it has been validated through simulations and experimental part.

It has been shown that the application of fractional order calculus poses some challenges when it comes to its implementation. This is due to the intrinsic character of the fractional (non-linear) exponent. Therefore, five mathematical existing approaches of the non-rational form of the fractional operator have been explored. These mathematical approximations have been compared, selecting the Chareff's approach as the one that better suits the frequency behaviour of the original transfer function with the fractional term. Moreover, a thorough tuning methodology has been proposed, analysing the robustness and also highlighting the capabilities and weaknesses of this new formulation. The fractional-resonant controller reduces the inter-harmonic excitation that conventional multi-harmonic proportional-resonant controller presents, and it extends its tracking capability to a wider frequency 

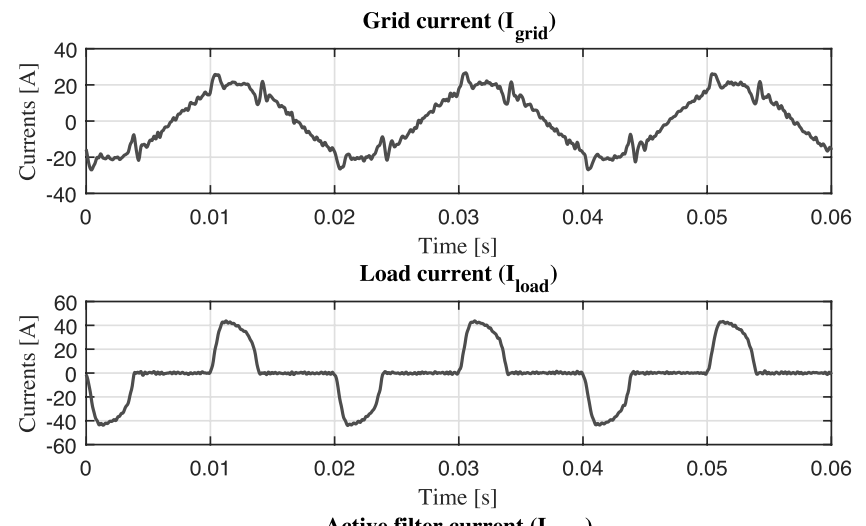

Active filter current $\left(I_{\text {conv }}\right)$

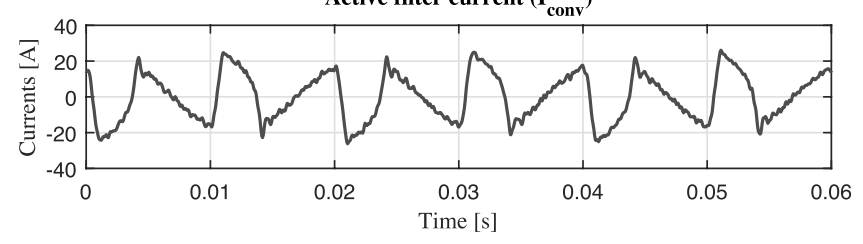

(a) Current waveforms
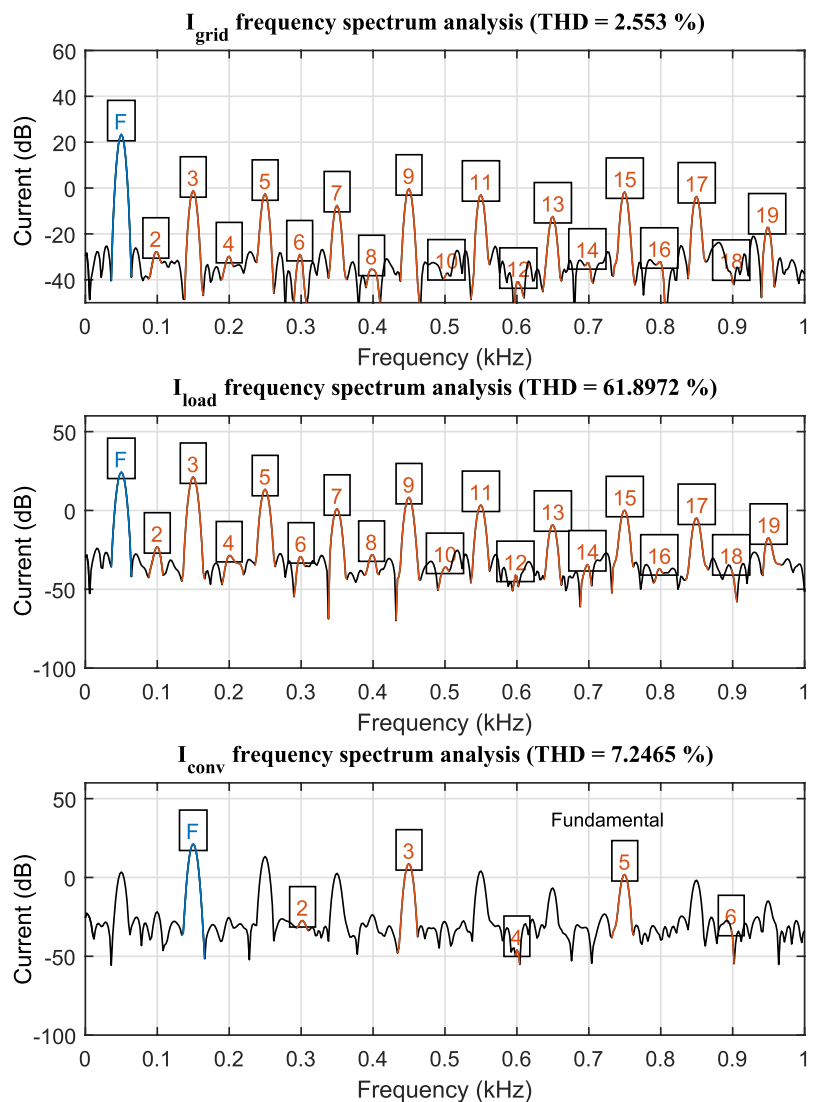

(b) Harmonic content distribution and THD

Fig. 25. Experimental results obtained operating as an active filter for the grid current $\left(\mathrm{I}_{\text {grid }}\right)$, the consumed load current $\left(\mathrm{I}_{\text {load }}\right)$ and the inverter injected current

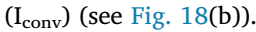



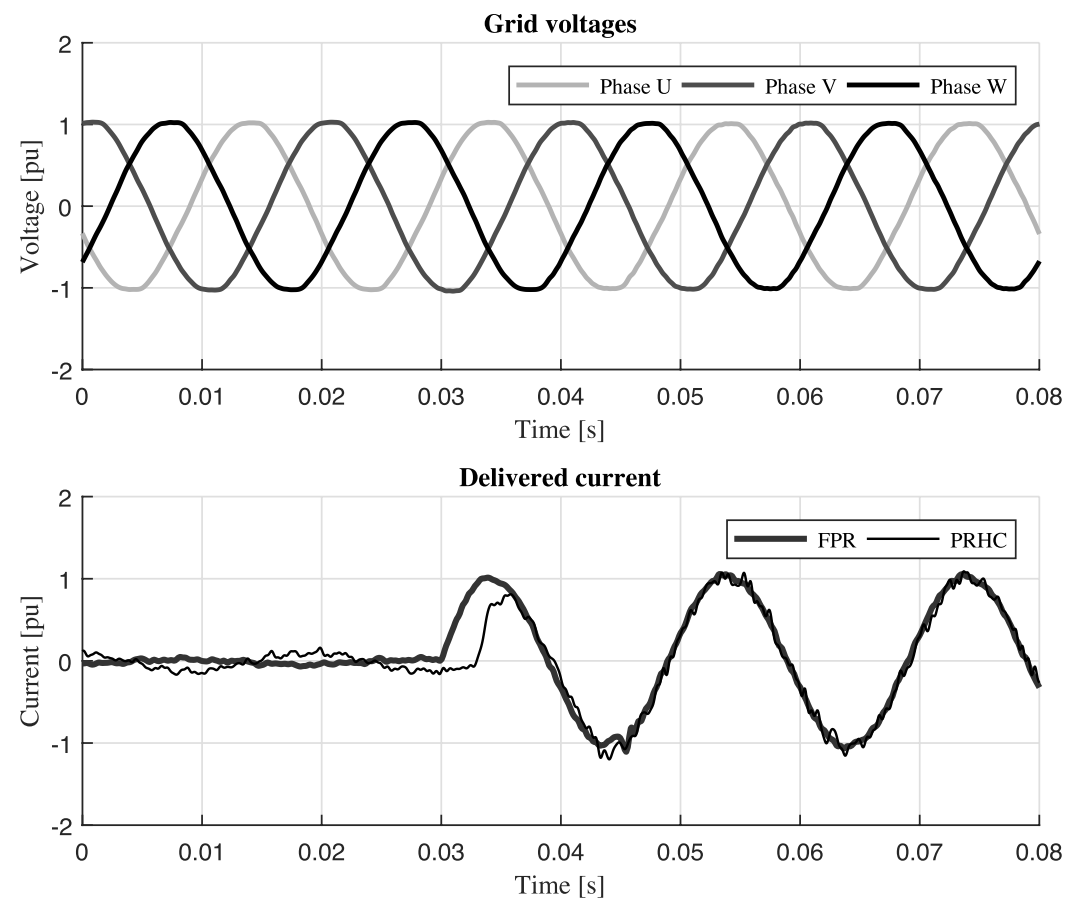

(a) Voltage and current waveforms
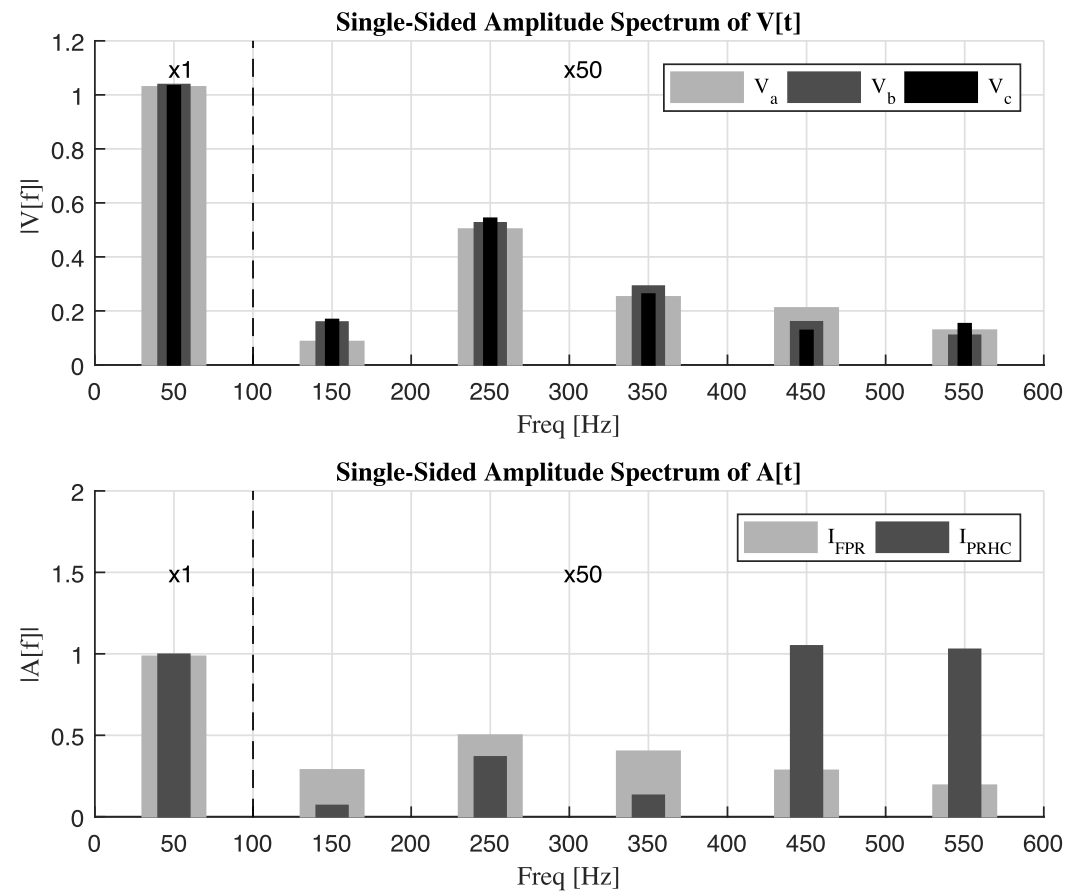

(b) Voltage and current decomposition in harmonic components

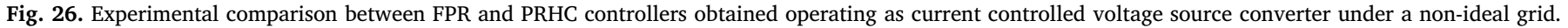

range. The results suggest the idea that an empirical fractional coefficient value around 1.5 could yield, in general, a better response for active filtering VSC applications compared to the conventional proportional-resonant controller.
The proposed controller has been implemented in a laboratory setup and its multi-harmonic tracking capability is demonstrated under three different conditions during the operation of a four-wire inverter: operating in an ideal scenario where the inverter's output is short- 
Table 3

Controller type comparison. Execution time and program memory data.

\begin{tabular}{lll}
\hline & Execution time & Program memory \\
\hline PI & $1.373 \mu \mathrm{s}$ & 135 bytes \\
PR & $0.495 \mu \mathrm{s}$ & 74 bytes \\
HC & $0.2325 \mu \mathrm{s}$ & 39 bytes \\
PRHC (3 HC) & $1.1925 \mu \mathrm{s}$ & 191 bytes \\
FPR & $0.9875 \mu \mathrm{s}$ & 150 bytes \\
\hline
\end{tabular}

circuited (balanced case), and when grid-connected. The grid-connected outline is evaluated operating as an active filter (delivering harmonic and inter-harmonic components) in which a strong non-linear load is connected on the AC side, and just delivering fundamental components in lightly distorted grids. In any case, the FPR has shown superior tracking behaviours for high harmonics, or practically equivalent ones for those cases in which harmonic compensators are employed, than other conventional controllers such as PRHCs.

\section{Appendix}

The computational burden in terms of memory program and execution time for the four controllers presented in Section 4.3 is shown in Table 3.

The micro-controller used is a Texas Instruments DSP, model Concerto F28M35. The base frequency clock used is $150 \mathrm{MHz}$. The software environment used to compile and execute the code is the Code Composer Studio version 6.1.1 from Texas Instruments and the compiler version is the TI v17.9.0.STS.

The execution and program memory refer to a single phase. However, the PI controller is exceptional due to the mathematical transformations required to operate in a synchronous reference frame. In fact, the PI case takes into account the execution time and the program memory needed for the calculation of a phase-locked loop (PLL), and the Park transformation.

\section{References}

[1] S. Buso, L. Malesani, P. Mattavelli, Comparison of current control techniques for active filter applications, IEEE Trans. Ind. Electron. 45 (5) (1998) 722-729, https:// doi.org/10.1109/41.720328.

[2] F.P. Dawson, A robust digital current control method for active power filters, IEEE Trans. Ind. Appl. 36 (4) (2000) 1158-1164.

[3] L.R. Limongi, R. Bojoi, G. Griva, A. Tenconi, Digital current-control schemes, IEEE Ind. Electron. Mag. 3 (1) (2009) 20-31, https://doi.org/10.1109/MIE.2009. 931894.

[4] A.G. Yepes, A. Vidal, J. Malvar, O. Lopez, J. Doval-Gandoy, Tuning method aimed at optimized settling time and overshoot for synchronous proportional-integral current control in electric machines, IEEE Trans. Power Electron. 29 (6) (2014) 3041-3054, https://doi.org/10.1109/TPEL.2013.2276059.

[5] A.R. Monter, E.J. Bueno, A. García-Cerrada, F.J. Rodríguez, F.M. Sánchez, Detailed analysis of the implementation of frequency-adaptive resonant and repetitive current controllers for grid-connected converters, Electr. Power Syst. Res. 116 (2014) 231-242, https://doi.org/10.1016/j.epsr.2014.06.011 URL: http://www. sciencedirect.com/science/article/pii/S037877961400220X.

[6] T.N. Nguyen, A. Luo, M. Li, A simple and robust method for designing a multi-loop controller for three-phase $\{\mathrm{VSI}\}$ with an lcl-filter under uncertain system parameters, Electr. Power Syst. Res. 117 (2014) 94-103, https://doi.org/10.1016/j. epsr.2014.08.011 URL: http://www.sciencedirect.com/science/article/pii/ S0378779614003083.

[7] X. Quan, X. Dou, Z. Wu, M. Hu, J. Yuan, Harmonic voltage resonant compensation control of a three-phase inverter for battery energy storage systems applied in isolated microgrid, Electr. Power Syst. Res. 131 (2016) 205-217, https://doi.org/ 10.1016/j.epsr.2015.10.010 URL: http://www.sciencedirect.com/science/article/ pii/S0378779615003065.

[8] L. Angel, J. Viola, M. Paez, Speed control of a motor-generator system using internal model control techniques, 2017 IEEE 3rd Colombian Conference on Automatic Control (CCAC) (2017) 1-6, https://doi.org/10.1109/CCAC.2017.8276453.

[9] R. Abassi, S. Chebbi, Energy management strategy for a grid-connected wind-solar hybrid system with battery storage: policy for optimizing conventional energy generation, Int. Rev. Electr. Eng. 7 (2012) 3979-3990.

[10] R. Abbassi, S. Saidi, S. Saidi, M. Hammami, S. Chebbi, Analysis of renewable energy power systems. Reliability and flexibility during unbalanced network fault, Handbook of Research on Advanced Intelligent Control Engineering and Automation 1 (2015) 651-686.

[11] R. Abbassi, S. Marrouchi, S. Saidi, A. Abbassi, S. Chebbi, Optimal energy management strategy and novel control approach for DPGSs under unbalanced grid faults, J. Circuits Syst. Comput. (2018).

[12] P. Mattavelli, Synchronous-frame harmonic control for high-performance ac power supplies, IEEE Trans. Ind. Appl. (2018).

[13] S. Fukuda, T. Yoda, A novel current-tracking method for active filters based on a sinusoidal internal model, IEEE Trans. Ind. Appl. 37 (3) (2001) 888-895, https:// doi.org/10.1109/28.924772.

[14] X. Yuan, W. Merk, H. Stemmler, J. Allmeling, Stationary-frame generalized integrators for current control of active power filters with zero steady-state error for current harmonics of concern under unbalanced and distorted operating conditions, IEEE Trans. Ind. Appl. 38 (2) (2002) 523-532, https://doi.org/10.1109/28.993175.

[15] D.N. Zmood, D.G. Holmes, Stationary frame current regulation of PWM inverters with zero steady-state error, IEEE Trans. Power Electron. 18 (3) (2003) 814-822, https://doi.org/10.1109/TPEL.2003.810852.

[16] A. Dell'Aquila, M. Liserre, V.G. Monopoli, P. Rotondo, Overview of PI-based solutions for the control of DC buses of a single-phase H-bridge multilevel active rectifier, IEEE Trans. Ind. Appl. 44 (3) (2008) 857-866, https://doi.org/10.1109/TIA. 2008.921405.

[17] C. Lascu, L. Asiminoaei, I. Boldea, F. Blaabjerg, Frequency response analysis of current controllers for selective harmonic compensation in active power filters, IEEE Trans. Ind. Electron. 56 (2) (2009) 337-347, https://doi.org/10.1109/TIE. 2008.2006953.

[18] A.G. Yepes, F.D. Freijedo, O. López, J. Doval-Gandoy, High-performance digital resonant controllers implemented with two integrators, IEEE Trans. Power Electron. 26 (2) (2011) 563-576, https://doi.org/10.1109/TPEL.2010.2066290.

[19] A. Timbus, M. Liserre, R. Teodorescu, P. Rodriguez, F. Blaabjerg, Evaluation of current controllers for distributed power generation systems, IEEE Trans. Power Electron. 24 (3) (2009) 654-664, https://doi.org/10.1109/TPEL.2009.2012527.

[20] C.S.S.D. Zammit, M. Apap, Comparison between PI and PR current controllers in grid connected PV inverters, Int. J. Electr. Electron. Sci. Eng. (2018).

[21] A.G. Yepes, F.D. Freijedo, J. Doval-Gandoy, O. López, J. Malvar, P. FernándezComesaña, Effects of discretization methods on the performance of resonant controllers, IEEE Trans. Power Electron. 25 (7) (2010) 1692-1712, https://doi.org/10. 1109/TPEL.2010.2041256.

[22] F. Wang, M.C. Benhabib, J.L. Duarte, M.A. Hendrix, Sequence-decoupled resonant controller for three-phase grid-connected inverters, 24th Annual IEEE Applied Power Electronics Conference and Exposition (2009) 121-127.

[23] F. Briz, M.W. Degner, R.D. Lorenz, Analysis and design of current regulators using complex vectors, IEEE Trans. Ind. Appl. 36 (3) (2000) 817-825, https://doi.org/10. $1109 / 28.845057$.

[24] C. Lascu, L. Asiminoaei, I. Boldea, F. Blaabjerg, High performance current controller for selective harmonic compensation in active power filters, IEEE Trans. Power Electron. 22 (5) (2007) 1826-1835, https://doi.org/10.1109/TPEL.2007.904060.

[25] R. Bojoi, G. Griva, M. Guerriero, F. Farina, F. Profumo, V. Bostan, Improved current control strategy for power conditioners using sinusoidal signal integrators in synchronous reference frame, IEEE 35th Annual Conference PESC vol. 6, (2004) 4623-4629, https://doi.org/10.1109/PESC.2004.1354817.

[26] I. Podlubny, Fractional Differential Equations, Academic Press, 1999.

[27] I. Podlubny, Fractional-order systems and PI-lambda-D-mu controllers, IEEE Trans. Autom. Control 44 (1) (1999) 208-214.

[28] C.A. Monje, B.M. Vinagre, V. Feliu, Y. Chen, Tuning and auto-tuning of fractional order controllers for industry applications, Control Eng. Pract. 16 (2008) 798812.

[29] Y. Luo, C. Wang, Y. Chen, Tuning fractional order proportional integral controllers for fractional order systems, Chinese Control and Decision Conference (2009).

[30] D.-J. Wang, X.-L. Gao, G.-J. Cai, Fractional-order optimization of PI-lambda-D-mu controllers for time-delay systems, Proceedings of 8th Asian Control Conference (2011).

[31] L. Mingqiu, J. Shuhua, Design of FO PI fractional order controller of ATP rough tracking system, 5th International Conference on Measuring Technology and 
Mechatronics Automation (2013) 442-445.

[32] R. Nazir, K. Zhou, N. Watson, A. Wood, Analysis and synthesis of fractional order repetitive control for power converters, Electr. Power Syst. Res. 124 (2015) 110-119, https://doi.org/10.1016/j.epsr.2015.03.005 URL: http://www. sciencedirect.com/science/article/pii/S0378779615000656.

[33] D. Heredero-Peris, E. Snchez-Snchez, C. Chilln-Antn, D. Montesinos-Miracle, S. Glceran-Arellno, A novel fractional proportional-resonant current controller for voltage source converters, 2016 18th European Conference on Power Electronics and Applications (EPE'16 ECCE Europe) (2016) 1-10, https://doi.org/10.1109/ EPE. 2016.7695305.

[34] F. Girbau-Llistuella, A. Sumper, D. Heredero-Peris, M.P. Gimnez, C.C. Anttn, J.A. Vidal-Clos, F. Daz-Gonzlez, R. Gallart-Fernndez, Demonstration and experience of the smart rural grid project, 2016 IEEE PES Innovative Smart Grid Technologies Conference Europe (ISGT-Europe) (2016) 1-6, https://doi.org/10.1109/ ISGTEurope.2016.7856257.

[35] P. Rodríguez, R. Teodorescu, I. Candela, A. Timbus, M. Liserre, F. Blaabjerg, New positive-sequence voltage detector for grid synchronization of power converters under faulty grid conditions, 37th IEEE Power Electronics Specialists Conference (2006).

[36] F.J. Rodríguez, E. Bueno, M. Aredes, L.G.B. Rolim, F.A.S. Neves, M.C. Cavalcanti, Discrete-time implementation of second order generalized integrators for grid converters, IEEE 34th Annual Conference IECON vol. 1, (2008) 176-181, https://
doi.org/10.1109/IECON.2008.4757948.

[37] E. Sánchez-Sánchez, D. Heredero-Peris, D. Montesinos-Miracle, Stability analysis of current and voltage resonant controllers for voltage source converters, 17th European Conference on Power Electronics and Applications (2015).

[38] R. Teodorescu, F. Blaabjerg, U. Borup, M. Liserre, A new control structure for gridconnected LCL PV inverters with zero steady-state error and selective harmonic compensation, 19th Annual IEEE Applied Power Electronics Conference and Exposition vol. 1, (2004) 580-586, https://doi.org/10.1109/APEC.2004.1295865.

[39] A.G. Yepes, F.D. Freijedo, O. López, J. Doval-Gandoy, Analysis and design of resonant current controllers for voltage-source converters by means of nyquist diagrams and sensitivity function, IEEE Trans. Ind. Electron. 58 (11) (2011) 5231-5250, https://doi.org/10.1109/TIE.2011.2126535.

[40] A. Charef, H. Sun, Y. Tsao, B. Onaral, Fractal system as represented by singularity function, IEEE Trans. Autom. Control 37 (9) (1992) 1465-1470, https://doi.org/10 1109/9.159595.

[41] B.M. Vinagre, I. Podlubny, A. Hernández, V. Feliu, Some approximations of fractional order operators used in control theory and applications, Fract. Calc. Appl. Anal. 3 (3) (2000) 231-248.

[42] A. Oustaloup, F. Levron, B. Mathieu, F. Nanot, Frequency-band complex noninteger differentiator characterization and synthesis, IEEE Trans. Circuits Syst. I: Fundam. Theory Appl. 47 (1) (2000) 25-39, https://doi.org/10.1109/81.817385. 